\title{
Narrative akustischer Heimsuchung um 1800 und heute: Hören und Erinnerung in Hoffmanns „Johannes Kreislers Lehrbrief“
}

Der Dokumentarfilm Alive Inside von Michael Rossato-Bennett, der 2014 auf dem Sundance Film Festival den World Cinema Jury Prize erhielt, erzählt, so der Untertitel, eine „Story of Music and Memory“. Er zeigt, wie demente Alzheimerpatienten durch Musik, die ihnen einst viel bedeutet hat, ,aufgeweckt“ werden können, sodass sie sich an ihr vergangenes Selbst, seine Vorlieben und Abneigungen, wieder lebhaft erinnern und sich sogar darüber unterhalten können. Das 2008 erschienene und von Washington Post, Chicago Tribune und Financial Times zum Best Book of the Year gewählte Buch Musicophilia des kürzlich verstorbenen Neuropsychiaters Oliver Sacks demonstriert, „what happens when music and the brain mix it up“ (Newsweek). ${ }^{1}$ Sacks erzählt Fallgeschichten von Patienten, die von Musikeinfällen heimgesucht werden, ohne diese „hint[s] from [the] unconscious“ entschlüsseln zu können, ${ }^{2}$ oder die mithilfe von Musik Zugang zu „emotions and associations“ finden, „that had been long forgotten, giving the patient access once again to moods and memories, thoughts and worlds that had seemingly been completely lost“. ${ }^{3}$ Die 2000 erschienene und bis 2011 jährlich neu aufgelegte Studie von Tia DeNora Music in Everyday Life behandelt „music as a technology of self“". Aus Befragungen von Probanden schließt sie, dass das Hören von „biographically key music“ nicht nur dem Nacherleben eines Ereignisses oder eines bedeutsamen Lebensabschnitts dient und die damit verbundenen Emotionen zurückbringt, sondern als solches auch der Selbstvergewisserung zuarbeitet: Musik als „prosthetic biography“. ${ }^{5}$

1 „FIRST to WORST: Books“, in: Newsweek 150.10 (3.9.2007), S. 60.

2 Oliver Sacks, Musicophilia: Tales of Music and the Brain, London 2007, S. 40.

3 Ebd., S. 380.

4 Tia DeNora, Music in Everyday Life, Cambridge 2010, S. 46.

5 Ebd., S. 63 u. 66. Dass Musik das kann, macht DeNora nicht zuletzt an ihrer temporalen Struktur fest: ,[M] usical structures may provide a grid or grammar for the temporal structures of emotional and embodies patterns as they were originally experienced“ (ebd., S. 68). Das vermutet auch Matussek, wenn er in der „Wiedererkennungsfunktion musikalischer Strukturen [...] und deren Subversion“ letztlich den Grund für déjà entendu-Effekte erkennt; Peter Matussek, „Déjàentendu. Zur historischen Anthropologie des erinnernden Hörens“, in: Günter Oesterle (Hg.), Déjà-vu in Literatur und bildender Kunst, München 2003, S. 289-309, hier S. 309. Vgl. ebenso jüngst 
Diese Beispiele zeigen, dass die Frage nach dem Zusammenhang von Musik und Erinnerung gegenwärtig im populärwissenschaftlichen Bereich auf großes Interesse stößt. Aus anderen Feldern ließe sich Ähnliches berichten, etwa aus der Philosophie. Philippe Lacoue-Labarthe stellt sich in „The Echo of the Subject“ schon 1979 die Frage: „What connection is there between autobiography and music? [...] What is it that ties together autobiography, that is to say, the autobiographical compulsion [...], and music - the haunting by music or the musical obsession?" ${ }^{\text {E }}$ Er ist seiner Zeit voraus. Andere Philosophen beschäftigen sich erst seit der Jahrtausendwende mit ähnlichen Themen. Jean Luc Nancy etwa formuliert in seiner phänomenologisch getränkten Studie „À l'écoute“ von 2002 die These: ,[L]auschen, das ist also in die Spannung und Obacht eines Selbstbezuges treten [...], [eines] Bezug[s], der ein ,Selbst‘ bildet oder ein ,zu sich‘ überhaupt. [...] [D]as Hören [erscheint] uns [...] als die Wirklichkeit dieses Zugangs [zum Selbst]“. ${ }^{7}$ Esoterischer geht es bei Peter Sloterdijk zu, der sich in seinem Essay „Klangwelt“ von $2007 \mathrm{zu}$ der These versteigt, über das Musikhören würde das Dasein im Mutterleib erinnernd nacherlebt. Der Fötus sei im Mutterleib „in ein internes sonores Kontinuum eingebettet“, sodass alles spätere Hören von Musik „immer auch das Register der tiefen Regressionen“ anspreche: „Musik [vermag] noch im erwachsenen, von der Härte des Realen geprägten Subjekt seine intime Vorgeschichte zu evozieren“. ${ }^{8}$

Man könnte über jede einzelne dieser Studien ein eigenes Buchkapitel schreiben; ${ }^{9}$ man könnte sich auch der Frage widmen, warum gerade in den letzten ca. 15 Jahren ein solches Interesse an Musik und Erinnerung aufgeflammt

Kai Preuß, der schreibt: „[Musik vermag] das Erinnerte in einer, wenn nicht der wesentlichen Eigenschaft anzusprechen: als etwas Zeitliches“; Kai Preuß, „Erinnerung und Zeitlichkeit“, in: Lena Nieper u. Julian Schmitz (Hg.), Musik als Medium der Erinnerung. Gedächtnis - Geschichte Gegenwart, Bielefeld 2016, S. 39-50, hier S. 39.

6 Philippe Lacoue-Labarthe, „The Echo of the Subject“, in: Lacoue-Labarthe, Typography: Mimesis, Philosophy, Politics, hg. v. Christopher Fynsk, Cambridge/London 1989, S.139-207, hier S. 140. Da es nach meiner Kenntnis keine deutsche Übersetzung gibt, habe ich hier der besseren Lesbarkeit halber auf die englische zurückgegriffen.

7 Jean Luc Nancy, Zum Gehör, übers. v. Esther von der Osten, Zürich/Berlin 2010, S. 20-22.

8 Peter Sloterdijk, „Klangwelt“, in: Sloterdijk, Der ästhetische Imperativ. Schriften zur Kunst, hg. v. Peter Weibel, Berlin 2007, S. 8-82, hier S. 10 f. Vgl. zu meiner Kritik an dieser und ähnlichen Positionen Nicola Gess, „Ideologies of Sound: Longing for Presence from the Eighteenth Century until Today“, in: Journal for Sonic Studies 10 (2015); online unter: https://www.researchcatalogue.net/view/220291/220292 (22.2.2017).

9 Vgl. zu psychologischen Studien den Beitrag von Manuela Schwartz zur Musiktherapie im vorliegenden Band, in dem sie allerdings nicht auf die musiktherapeutische Kur von Demenzerkrankungen eingeht. 
ist. Doch möchte ich in diesem Artikel die aktuellen Studien lediglich als Sprungbrett nutzen, um über die literarische Vorgeschichte dieses Trends zu sprechen. ${ }^{10}$

Dass es eine solche Vorgeschichte gibt, scheint den meisten der erwähnten Studien durchaus bewusst zu sein. Lacoue-Labarthe beginnt mit Hölderlin und Mallarmé, greift für die Motti seiner Kapitel u. a. auf Valéry, Schiller, Tieck, Schlegel, Arnim/Brentano und Rückert zurück, und endet mit einem Gedicht von Wallace Stevens, in dem es $u$. a. heißt: „The self is a cloister full of remembered sounds“. ${ }^{11}$ Nancy zitiert Valéry und Wagner und integriert zudem ein eigenes Lautgedicht. ${ }^{12}$ Da Lacoue-Labarthe und Nancy die Grenzziehung zwischen Literatur und Philosophie nicht akzeptieren, ist ihre Bezugnahme auf literarische Texte vielleicht noch nicht besonders bemerkenswert. Doch beziehen sich auch die psychologischen Studien von Sacks und DeNora auf Literatur, indem sie en passant den Namen Prousts und damit dessen Roman À la recherche du temps perdu bzw. das Konzept der mémoire involontaire anführen: „[F]amiliar music acts as a sort of Proustian mnemonic, eliciting emotions and associations that

10 Mit Bezugnahme auf einige Filmszenen (wie Veronika Voss' Performance des Songs Memories Are Made of This in Fassbinders Die Sehnsucht der Veronika Voss [BRD 1982] oder auch, ein klassisches Beispiel, Ilsa Lunds „Play it, Sam“ in Curtiz' Casablanca [USA 1942] in Bezug auf den Song As Time Goes By, den Sam dann auch tatsächlich spielt) machte mich Mary Helen Dupree darauf aufmerksam, dass ein wichtiger Grund für diesen Trend auch in bestimmten filmischen Verfahren liegen könnte, die Musik als Erinnerungsmarker einsetzen und etwa als Auslöser für narrative flash backs verwenden. Zugleich ist jedoch wichtig zu berücksichtigen, dass der Film hier seinerseits bereits auf ältere Strategien des Musiktheaters rekurriert, über musikalische Motive (,Erinnerungsmotive‘, ,Leitmotive`) dem Hörer und bisweilen auch den Bühnenfiguren bestimmte Personen oder Ereignisse wieder in Erinnerung zu rufen (vgl. dazu u. a. Nicola Gess, „,Hoffmanns Erzählungen“ erzählen, oder: Die Oper als Erzählung“, in: Pascal Nicklas [Hg.], Literatur und Musik, Berlin [in Vorbereitung]). Verbunden werden solche Strategien insbesondere mit den Musikdramen Wagners, und nicht von ungefähr finden sich etwa in der empirischen Psychologie um 1900 immer wieder Assoziationen zu Wagners Leitmotivik, wenn von Musik und Erinnerung die Rede ist (z. B. bei Sándor Ferenczi, „Zur Deutung einfallender Melodien“, in: Ferenczi, Bausteine zur Psychoanalyse, Bd. III: Arbeiten aus den Jahren 1908-1933, 2., unveränd. Aufl., Bern/Stuttgart/Wien 1964 [ND der Ausg. 1938], S. 23-25, hier S. 24; oder bei Sigmund Exner, Entwurf zu einer physiologischen Erklärung der psychischen Erscheinungen [1894], Frankfurt a. M. 1999, S. 317). Wagner aber ist seinerseits in seiner Konzipierung von Musik als Stimme der Erinnerung/des Unbewussten maßgeblich von romantischen Konzeptionen beeinflusst, wie sie im vorliegenden Artikel behandelt werden.

11 Lacoue-Labarthe, „The Echo of the Subject“, S. 207.

12 Auf den ersten Seiten des Kapitels „Interludium. Stumme Musik“ (Nancy, Zum Gehör, S. 3334), verfasst ursprünglich für das 2000 erschienene Buch $\mathrm{Mmmmmmm}$ der Künstlerin Susanna Fritscher. 
had been long forgotten“; ${ }^{13}$ „music [...] is a mediator of, in Proust's sense, the aesthetic, memory-encrusted unconscious““. ${ }^{14}$

Aber bevor der literarische Faden aufgenommen wird, soll noch auf eine weitere Nuance des gegenwärtigen Zusammendenkens von Musik und Erinnerung hingewiesen werden. In den Fallgeschichten der erwähnten Studien wird die musikalische Auslösung einer Erinnerung von den Betroffenen in der Regel positiv valorisiert. Sacks und Lacoue-Labarthe sprechen hingegen auch von einer Heimsuchung, wenn sie den Charakter der mit Erinnerung beladenen Musik beschreiben wollen. ${ }^{15}$ Sie verwenden den Terminus des ,musical haunting und berufen sich dabei auf Theodor Reiks autobiographisch geprägte Studie Haunting Melody (1953), in der der Psychoanalytiker zu begreifen versucht, warum er in den Wochen nach dem Tod seines Lehrers und Freundes Karl Abraham von einer Melodie aus dem letzten Satz der zweiten Sinfonie Gustav Mahlers heimgesucht wurde. ${ }^{16}$ Reik schreibt über diese Erfahrung:

I seemed to hear the ghostlike onset of the choir, the voices, [...], first misterioso, solemnly. [...] [I]t began to haunt me whenever I thought of Dr. Abraham. [...] But it also interfered with other trains of thought which had nothing to do with him. [...] It haunted me from that evening until New Year's day, and rarely left me for more than an hour. It was as if that melody had thrown a spell over me. I could not get rid of it, however much I tried to shake it off. ${ }^{17}$

Die Erinnerung an Mahlers Choral ist mit der Erinnerung an einen Toten verbunden, der sich über die Musik immer wieder in die Gedanken des Betroffenen drängt. Passenderweise handelt es sich bei dem Choral um eine Totenklage bzw. Auferstehungsmusik, die zudem bereits als Chor von Geisterstimmen (,ghost-

13 Sacks, Musicophilia, S. 380; vgl. auch ebd., S. 40.

14 DeNora, Music in Everyday Life, S. 68.

15 Auch der so häufig referierte Proust macht von dieser Metapher Gebrauch, wenn er „das kleine Thema [der Sonate de Vinteuil, N. G.]“ als einen „gefangenen Geist“ beschreibt, der von den Instrumenten heraufbeschworen wird, um in Swann die unwillkürliche Erinnerung an seine verlorene Geliebte auszulösen. So wohltuend dieser Spuk letztlich für Swann ist, indem er ihn zur nachträglichen Trauerarbeit zwingt, so schmerzvoll ist er doch zunächst: „Plötzlich aber war es, als sei sie eingetreten, und diese Erscheinung bereitete ihm einen Schmerz, der ihn so reißend durchfuhr, daß er die Hand an sein Herz führen mußte. [...] [A]lle seine Erinnerungen [...], die er bis zu diesem Tag unsichtbar in den Tiefen seines Innern zurückzuhalten vermocht hatte, [...] [waren] aufgewacht“; Marcel Proust, Werke, Abt. 2: Auf der Suche nach der verlorenen Zeit, Bd. 1: Unterwegs zu Swann, übers. von Eva Rechel-Mertens, Frankfurt a. M. 1994, S. 503 u. 499.

16 Vgl. Theodor Reik, The Haunting Melody: Psychoanalytic Experiences in Life and Music, New York 1953.

17 Ebd., S. $221 \mathrm{f}$. 
like“ und „misterioso“) vertont wurde. Doch zur quälenden Heimsuchung wird die erinnerte Musik erst dadurch, dass sie Reik gegen seinen Willen, in unpassendsten Momenten aufsucht und bei seinen alltäglichen Verrichtungen stört und dass sie ihm etwas sagen zu wollen scheint, das er nicht entschlüsseln kann: „I pondered about what the motif wanted to convey to me. I heard its message, but I did not understand it; it was as if it had been expressed in a foreign language I did not speak“ ${ }^{18}$ Als Freud-Schüler geht Reik davon aus, dass Spukphänomene Ausdruck der Wiederkehr eines Verdrängten sowie in aller Regel auch eines Schuldkomplexes sind. Das ,haunting، durch den Choral Mahlers interpretiert er darum als Symptom: Als Geisterstimme weist sie ihn auf etwas hin, das er verdrängt hat und das mit seinem toten Lehrer und Freund Karl Abraham zu tun haben muss. In seiner jahrzehntelangen Selbstanalyse gelangt Reik dann wenig überraschend zu dem Schluss, dass ein ödipaler Schuldkomplex (der uneingestandene Wunsch, Abrahams Position einnehmen zu können) der Grund für die musikalische Heimsuchung war.

Reiks Studie ist interessant für das hier $\mathrm{zu}$ verhandelnde Thema, weil sie zeigt, dass es eine dunkle Kehrseite des musikalischen Aufrufens von Erinnerungen zu geben scheint, die mit dem traumatischen Charakter des vergangenen Erlebnisses $\mathrm{zu}$ tun hat. Und sie greift, um diese Kehrseite $\mathrm{zu}$ verbalisieren, zu einem Spuknarrativ, das durch die Aufnahme bei Lacoue-Labarthe und Sacks auch gegenwärtig wieder Konjunktur im Zusammendenken von Musik und Erinnerung hat. ${ }^{19}$

Eine literarische Urszene zu diesem Narrativ hat Cathy Caruth in Unclaimed Experience: Trauma, Narrative, and History (1996) ausgemacht. ${ }^{20}$ Sie weist darauf hin, dass Sigmund Freud in Jenseits des Lustprinzips den traumatischen Wiederholungszwang mit einer Passage aus Torquato Tassos Epos Gerusalemme liberata (1581) illustriert, in der ebenfalls die akustische Heimsuchung, hier durch die Stimme der Geliebten, eine zentrale Rolle spielt. Der Held Tancredi hat, ohne es zu wollen, seine Geliebte Clorinda im Kampf getötet; nach ihrem Tod verwundet er sie unwissentlich ein zweites Mal, indem er eine Zypresse verletzt, in die die Seele Clorindas eingewandert war. Erst als aus dem Baum eine klagende Stimme

18 Ebd., S. 223.

19 Vgl. für ein Pendant in der Popkultur etwa auch das zu Reik in etwa zeitgenössische screen printing von Roy Lichtenstein, The Melody Haunts My Reverie (1965).

20 Vgl. Cathy Caruth, Unclaimed Experience: Trauma, Narrative, and History, Baltimore 1996, S.1f. Den Hinweis auf Caruth verdanke ich Philipp Schweighauser. Weitere Ur-Szenen könnte man in antiken Mythen, wie vor allem dem Echo-Mythos finden, in dem die von Narziss verschmähte Nymphe Echo in einer Höhle verkümmert, sodass nur noch die Stimme von ihr übrig bleibt. 
ertönt, mit der sich Clorinda zu erkennen gibt - „Du hast aus meinem Körper mich vertrieben [...]. Warum zerstörst du nun den armen Stamm, / In den mein hartes Schicksal mich gebannt hat? / So willst du, Unbarmherz'ger, deine Feinde / Auch nach dem Tode noch im Grabe kränken? // Clorinda war ich [...]“21 -, erkennt Tancredi sein doppeltes Verbrechen und flieht vor der geisterhaften Mahnung an seine Taten. Während Freud, andernorts durchaus hellhörig für stimmliche Symptome, diesem Detail keine weitere Aufmerksamkeit schenkt, hebt Caruth den Zusammenhang hervor, den Tassos Epos zwischen der traumatischen Erfahrung und der akusmatischen Stimme knüpft:

The voice of his beloved addresses him and [...] bears witness to the past he has unwittingly repeated. [...] Tancred's story thus represents traumatic experience [...] as the enigma of the otherness of a human voice that cries out from the wound, a voice that witnesses a truth that Tancred himself cannot fully know. ${ }^{22}$

Ich möchte diese Hinweise zum Anlass nehmen, um die literarische Vorgeschichte des Zusammendenkens von Musik und (traumatischer) Erinnerung näher zu beleuchten. Hör-Wissen soll hier also erstens in dem Sinne verstanden werden, dass es um ein vergessenes/verdrängtes Wissen von der Vergangenheit geht, dessen Erinnerung durch Musik oder andere klangliche Phänomene bei ihren Hörern wiederbelebt werden kann. ${ }^{23}$ Zweitens soll das Augenmerk dabei auf die

21 Torquato Tasso, „Die Befreiung Jerusalems“, in: Tasso, Werke und Briefe, übers. u. eingel. v. Emil Staiger, München 1978, S. 183-674, hier S. 486.

22 Caruth, Unclaimed Experience, S. 3.

23 Vgl. zum Begriff der ,Wiederbelebung Edgar Erdfelder, [Art.] „Wiederbelebte Erinnerung“, in: Nicolas Pethes u. Jens Ruchatz (Hg.), Gedächtnis und Erinnerung. Ein interdisziplinäres Lexikon, Reinbek 2001, S. 639. Erdfelder versteht darunter „wiedereinsetzende Erinnerungen an traumatische Ereignisse, die in einer amnestischen Phase zuvor nicht erinnert werden konnten“ (ebd.). Musik oder andere klangliche Phänomene werden von mir im vorliegenden Artikel zum einen als Trigger einer unwillkürlichen Erinnerung verstanden, zum anderen als Medium des Erinnerns, d. h. - nach Gudehus et al. - auf der „Schnittstelle von Gedächtnis und Erinnerung“ positioniert; vgl. Christian Gudehus, Ariane Eichenberg u. Harald Welzer (Hg.), Gedächtnis und Erinnerung. Ein interdisziplinäres Handbuch, Stuttgart 2010, S. 127 (allerdings wird von Gudehus et al. Musik selbst nicht als ein Medium des Erinnerns geführt). Die Begriffe ,Gedächtnis‘ und ,Erinnerung werden im vorliegenden Beitrag im Sinne der basalen Definition von Gedächtnis als „System zur Aufnahme, zur Aufbewahrung [...] jeder Art von Informationen“ und Erinnerung als „Abrufen“ dieser Informationen verwendet (ebd., S. vii). Außerdem ist mir in Bezug auf die Abgrenzung von Gedächtnis und Erinnerung um 1800 die zeitgenössische Unterscheidung Jean Pauls wichtig: „Er unterschied Gedächtnis und Erinnerung durch ihre differierenden Ordnungsstrukturen des räumlichen Nebeneinanders und des zeitlichen Nacheinanders [...]. Jean Paul band diese Unterscheidung an ein Verständnis von Erinnerung als Tätigkeit, während er das Gedächtnis ,ein 
Geschichte dieses Hör-Wissens und, genauer, auf den literarischen Diskurs um dieses Hör-Wissen in den Jahren um 1800 gelegt werden, aus dem vor allem die Erzählung einer akustischen Heimsuchung von E. T. A. Hoffmann herausgegriffen wird. ${ }^{24}$ Diese Konzentration auf den literarischen Diskurs nehme ich aus drei hier nur thesenartig formulierbaren Gründen vor, von denen der dritte im weiteren Verlauf des Artikels ausgearbeitet wird.

a) Das Hör-Wissen, von dem hier die Rede ist, ist auf Narrativierung angewiesen. Nicht nur, weil seine Darstellung über die Erzählung (zum Beispiel von Fallgeschichten, die sich um 1800 ebenso häufig in der entstehenden Psychologie wie in der Dichtung finden und wissenschaftlichen wie literarischen Ansprüchen Genüge zu tun suchen) ${ }^{25}$ funktioniert; das ist übrigens auch in den eingangs genannten Werken von Rossato-Bennett, Sacks und DeNora so, die eine Fallgeschichte an die nächste reihen. Sondern auch, weil die vergessene/ verdrängte Erinnerung, die in diesen Erzählungen durch Musik oder andere quasi-musikalische Phänomene aufgerufen wird, der Narrativierung bedarf. Diese Notwendigkeit ist insbesondere in der Trauma-Literatur wiederholt herausgearbeitet worden: Das traumatische Ereignis entzieht sich der bewussten Erinnerung ebenso wie der sprachlichen Darstellung, ist aber gerade deswegen auf seine nachträgliche Narrativierung angewiesen, weil es nur so aufgearbeitet und in die autobiographische oder auch kollektive Selbsterzählung integriert werden kann. ${ }^{26}$

b) Um 1800 entsteht nicht nur das Konzept des Unbewussten, das seine wohl eindrücklichste Formulierung in literarischen Texten wie denen E. T. A. Hoff-

nur aufnehmendes, nicht schaffendes Vermögen“ nannte“ (Günter Oesterle, „Erinnerung in der Romantik. Einleitung“, in: Oesterle [Hg.], Erinnern und Vergessen in der europäischen Romantik, Würzburg 2001, S. 7-23, hier S. 12).

24 Die Untersuchung von Narrativen akustischer Heimsuchung wird hier also nur in einem sehr beschränkten Umfang vorgenommen; sie könnte stark ausgeweitet werden, z. B. auf akustische Heimsuchungen im Kontext des Spiritismus (vgl. dazu etwa Leigh Eric Schmidt, Hearing Things: Religion, Illusion, and the American Enlightenment, Cambridge 2002) und ihren Niederschlag in der Literatur oder auch auf die Rezeption neuer akustischer Medien, die in den literarischen Texten des 19. bis frühen 20. Jahrhunderts häufig als Geister-Medien beschrieben werden.

25 Vgl. zur Fallgeschichte um 1800 u. a. Nicolas Pethes, Literarische Fallgeschichten. Zur Poetik einer epistemischen Schreibweise, Konstanz 2016.

26 Vgl. dazu z. B. Gabriele Rippl et al. (Hg.), Haunted Narratives: Life Writing in an Age of Trau$m a$, Toronto 2013. Inzwischen ist in der Forschung zu Gedächtnis und Erinnerung allgemein akzeptiert, dass nicht erst die Artikulation von Erinnerung narrativ organisiert ist, sondern auch die strukturelle Ordnung der Erinnerung selbst; vgl. J. Straub u. W. Ernst, [Art.] „Narration“, in: Nicolas Pethes u. Jens Ruchatz (Hg.), Gedächtnis und Erinnerung. Ein interdisziplinäres Lexikon, Reinbek 2001, S. 399-405. 
manns findet, in denen vor allem die unheimliche Wiederkehr des Verdrängten thematisiert wird und auf die auch Freud später in seiner Theoriebildung zurückgreifen wird. Sondern um diese Zeit entsteht auch das Konzept einer besonderen Wirkungsmacht der Musik, das sich unter anderem aus der Überzeugung eines unmittelbaren, nicht zuletzt auch physiologisch begründeten Zusammenhangs von Klangkunst und Seelenleben herleitet. ${ }^{27}$ Musik als Sprache des Unbewussten - diese schon bald zum common place herabsinkende, von Psychologen, Musikphilosophen und Schriftstellern geteilte Annahme war um 1800 so neu wie literarisch produktiv. Zugleich ist es prägend für die Literatur um 1800, ein neues Konzept von Erinnerung beschrieben und mitentwickelt $\mathrm{zu}$ haben, das deren Unwillkürlichkeit und Partikularität betont: „[D]ie Darstellung schreckhaften Auftauchens zusammenhangloser Bruchstücke, unvermittelter flash backs, wie sie die Traumaforschung heute analysiert, ist erst in der Romantik poesiefähig geworden“, so Günter Oesterle zusammenfassend in der Einleitung zu Erinnern und Vergessen in der europäischen Romantik. ${ }^{28}$ Damit ändert sich aber auch das Metaphernfeld der Erinnerung, in dem die „traditionelle Gedächtnismetaphorik von Magazin und Wachstafel“ sowie des „Augensinns als bislang privilegiertem Erinnerungsträger“ durch ein „raumzeitliches Intermedium“, wie zum Beispiel die Musik, und „andere, niedere Sinne als Erinnerungsstimuli“, wie zum Beispiel den Hörsinn, abgelöst werden. ${ }^{29}$

c) Literatur um 1800 interessiert sich in besonderer Weise für das hier diskutierte Hör-Wissen, weil es ihr eine Reflexion auf die eigenen medialen Bedingungen erlaubt. Dabei lassen sich zwei Positionen unterscheiden, die Hoffmanns Erzählung „Kreislers Lehrbrief“ souverän aufgreift und zugleich hinterfragt. Zum

27 Für eine Auseinandersetzung mit den Merkmalen und Gründen des Diskurses über die Wirkungsmacht von Musik vgl. Nicola Gess, Gewalt der Musik. Literatur und Musikkritik um 1800, 2. Aufl., Freiburg/Berlin 2011; Caroline Welsh, Hirnhöhlenpoetiken. Theorien zur Wahrnehmung in Wissenschaft, Ästhetik und Literatur um 1800, Freiburg 2003.

28 Oesterle, „Erinnerung in der Romantik. Einleitung“, S. 15.

29 Ebd., S. 9. Bettine Menke hat in ihrer Studie Prosopopoiia die große Bedeutung der rhetorischen Figur der Prosopopoiia für die romantische Literatur herausgestellt. Sie schreibt: „In der Formel ,Giving a Voice to the Voiceless ‘ wurde die Prosopopoiia als Figur für das Erinnern vorgeschlagen, das als eine Vergegenwärtigung des Abwesenden und Toten in der Stimme gedacht wird“. In der Auseinandersetzung mit den Texten zeigt sie dann aber vor allem die Dialektik von Erinnern und Vergessen auf; denn die Prosopopoiia macht „in der Restitution das Vergessen- und Verlorensein [...] vergessen. Die fantasmagorische fiktive Stimme der Texte und Stimme zugleich für die Texte verschleiert, indem sie in ihrer Fiktion ihre Rhetoriziät leugnet, die Abwesenheit und Stummheit, den Tod, die sie voraussetzt; und sie markiert diese - als Figur - noch in deren figurativer Verstellung“; Bettine Menke, Prosopopoiia. Stimme und Text bei Brentano, Hoffmann, Kleist und Kafka, München 2000, beide Zit. S. 260. 
einen besinnt sich Literatur um 1800 auf ihre eigenen ,musikalischen' Qualitäten und möchte selbst wieder als sonale Kunst verstanden und praktiziert werden. ${ }^{30}$ Angelehnt an die Sprachursprungstheorien der Zeit spielt dabei die Vorstellung, dass in den Klängen und Rhythmen der literarisch geformten Sprache die Stimmen einer längst vergangenen Vorzeit wieder laut werden können, eine zentrale Rolle. Eine meiner Hypothesen ist daher, dass Literatur, die sich um 1800 mit dem hier zu verhandelnden Hör-Wissen befasst, selbst als akusmatischer Klang verstanden werden möchte, der der unbekannten, dafür aber umso potenteren Vergangenheit eine unsichtbare Präsenz in der Gegenwart verschafft. ${ }^{31} \mathrm{Zum}$ anderen ist dieses Hör-Wissen, so eine weitere Hypothese, auch für das um 1800 entstehende Genre der Schauerliteratur von großer Bedeutung, ${ }^{32}$ weil es erstens als Stilmittel des Unheimlichen Verwendung findet, weil zweitens die referenzielle Unbestimmtheit, die die Tonkunst im Allgemeinen und den akusmatischen Klang im Besonderen auszeichnet, dort als Qualität der literarischen Beschreibung wertgeschätzt wird, und weil sich drittens auch die Schauerliteratur (Hoffmanns) selbst durch akusmatische Erzähler auszeichnet, die nicht nur das, wovon sie erzählen, im Dunkeln belassen, sondern auch selbst im Dunkeln bleiben.

\section{Ein ursprünglicher Gewaltakt}

E. T. A. Hoffmanns „Kreislers Lehrbrief“ (1815) enthält die Erzählung einer musikalischen Heimsuchung. In einem Wald liegt ein von rötlichen Moosen bewachsener Stein, der den jungen Komponisten Chrysostomus auf rätselhafte Weise

30 Vgl. den Beitrag von Mary Helen Dupree im vorliegenden Band.

31 Unter einem akusmatischen Klang verstehe ich mit Michel Chion (Audio-Vision: Sound on Screen, New York 1994), der den Begriff wiederum von Pierre Schaeffer (Traité des objets musicaux, Paris 1966) entlehnt, einen Klang, den man hört, ohne seine Quelle sehen zu können. Ursprünglich stammt der Begriff der Akusmatik aus dem Griechischen, wo er sich auf die Schüler des Pythagoras bezog, die der Lehre ihres Meisters lauschen mussten, während dieser hinter einem Vorhang stand; vgl. Mladen Dolar, His Master's Voice. Eine Theorie der Stimme, übers. v. Michael Adrian u. Bettina Engels, Frankfurt a. M. 2007, S. 82-84.

32 Dies lässt sich auch als Gegengewicht zu der in der Forschung mehrfach hervorgehobenen Bedeutung des Visuellen für die gothic novel verstehen. So schreibt etwa Robert Miles, dass man die gothic romance als eine „form of visual technology“ verstehen könne, die ,alongside the forerunners of modern cinema“ zu stellen sei; Robert Miles, „Introduction: Gothic Romance as Visual Technology“, in: Miles (Hg.), Gothic Technologies: Visuality in the Romantic Era. Romantic Circles: Praxis Series, 2005, online unter: https://www.rc.umd.edu/praxis/gothic/intro/miles. html. (22.2.2017). 
anzieht und aus dem die geisterhaften Gesänge einer jungen Frau zu tönen scheinen:

[J]ene Gesänge, die mich wie Geisterstimmen umtönten, wären in den Moosen des Steins [...] aufbewahrt [...]. Wirklich geschah es auch, daß den Stein betrachtend, ich [...] dann herrlichen Gesang des Fräuleins vernahm [...]. [...] [A]n den Stein gelehnt, hörte ich oft, wenn der Wind durch des Baumes Blätter rauschte, es wie holde herrliche Geisterstimmen ertönen $[\ldots . .]^{33}$

Schließlich materialisiert sich aus den Geisterstimmen tatsächlich der Geist eines Fräuleins:

Ich sah den Stein - seine roten Adern gingen auf wie dunkle Nelken, deren Düfte sichtbarlich in hellen tönenden Strahlen emporfuhren. In den langen anschwellenden Tönen der Nachtigall verdichteten sich die Strahlen zur Gestalt eines wundervollen Weibes, aber die Gestalt war wieder himmlische herrliche Musik! ${ }^{34}$

Die Rede von Geisterstimmen kann bei Hoffmann vielerlei implizieren: Sie kann darauf hinweisen, dass es um eine Stimme aus dem Innern des Hörers bzw. in der vorliegenden Erzählung um die akustische Imagination eines Komponisten geht; sie kann darauf hinweisen, dass es um Emanationen einer fremden Psyche geht, die vom Hörer Besitz ergreifen will - häufig ist hier an magnetistische bzw. mesmeristische Kontexte zu denken, wie sie in „Kreislers Lehrbrief“ durch den geheimnisvollen Fremden aufgerufen werden. Entscheidend für mich ist in diesem Zusammenhang jedoch eine dritte Implikation, die die Geisterstimmen als Stimmen der Toten bzw. als Mahnung an ein vergangenes Verbrechen versteht. Sie steht im Kontext zahlreicher anderer Erzählungen des späten 18. bis mittleren 19. Jahrhunderts, in denen auf ein - mehr oder weniger kapitales Verbrechen - die akustische Heimsuchung folgt, von Johann Wolfgang von Goethes „Die Sängerin Antonelli“ und „Das rätselhafte Klopfen“ aus dessen Novellensammlung Unterhaltungen deutscher Ausgewanderten (1795) bis zu Edgar Allan Poes „Tell-Tale Heart“ (1843) oder „The Fall of the House of Usher“ (1839), um nur einige Beispiele zu nennen.

Auch in Hoffmanns Erzählung ist der akustische Spuk durch einen Mord motiviert: Einst kam ein geheimnisvoller Fremder auf die Burg, der der Musiklehrer und Geliebte des Burgfräuleins wurde. Sie treffen sich nachts im Wald, um

33 E. T. A. Hoffmann, „Kreisleriana“, in: Hoffmann, Sämtliche Werke in 6 Bdn., Bd. 2.1: Fantasiestücke in Callot's Manier. Werke 1814, hg. v. Hartmut Steinecke, Frankfurt a. M. 1993, S. 32-455, hier S. $451 \mathrm{f}$.

34 Ebd., S. $452 \mathrm{f}$. 
zu musizieren: Sie singt, er spielt die Laute, und weithin hört man ihre ,schauerlich“ klingenden „Melodien“. ${ }^{35}$ Das Burgfräulein wird ermordet, vermutlich von dem verschwundenen Fremden. Man findet ihre Leiche nebst der zertrümmerten Laute verscharrt unter einem großen Stein, auf dem sie mit dem Fremden gesessen hatte und aus dem nun Blutstropfen quellen. Aus dem Blut wachsen später die Moose, und seit dieser Zeit „nistet alljährlich auf dem Baum eine Nachtigall und singt um Mitternacht in klagenden, das Innerste durchdringenden Weisen“. ${ }^{36}$

Freud hat, wie bereits erwähnt, nicht zuletzt im Rückgriff auf Erzählungen E. T. A. Hoffmanns Spukphänomene als Wiederkehr eines Verdrängten sowie auch als Ausdruck eines Schuldkomplexes interpretiert. Ohne auf diesen common place hier noch einmal eingehen zu müssen, liegt es nahe, die Geisterstimmen des „Lehrbriefs“ entsprechend zu deuten: Der Spuk im Wald gemahnt die Nachkommen des Rittergeschlechts an ein kollektives Trauma, das ungesühnte Verbrechen, das in der von Generation zu Generation weitergegebenen Erzählung als düstere Lücke (das Verbrechen wird nie aufgeklärt) präsent ist; als Geisterstimme kehrt die ermordete Sängerin zurück und betört ihren musikalischen Nachfahren durch ihren Gesang, so wie sie einst selbst durch den Gesang des musikalischen Fremden betört wurde. Chrysostomus hört die Stimme der ermordeten Sängerin; und der junge Kreisler, dem der alte Kreisler die Geschichte des Chrysostomus wiedergibt, hört die Stimme des in seinem „Innern versteckten Poeten“37 - eine Stimme, die von Gotthilf Heinrich von Schubert, auf den sich Hoffmann an dieser Stelle bezieht, in der Symbolik des Traums (1814) als „Stimme des Gewissens“ bezeichnet wird, die den Menschen im Traum ebenso wie in der „poetischen Begeisterung“ heimsuche. Eines der Beispiele, die Schubert dafür gibt, ist bezeichnenderweise „das Wimmern des Ermordeten“, das „Verbrecher“, „wachend und träumend“, „lange Jahre begleitet“. 38

Verglichen mit Hoffmanns anderen Musikerzählungen ist die blutige Drastik dieses Geschehens recht einmalig. Und sie ist auch beunruhigend, weil sie im Kontext eines Lehrbriefs steht, der den jungen Musiker über das Wesen der Musik und der Komposition aufklären soll: Der „Lehrbrief“ liefert also einen MusikEntstehungs-Mythos, der auf einen ursprünglichen Gewaltakt gegründet ist hier ist man wieder versucht, an Freuds Totem und Tabu (1913) zu denken. Sei es das Singen der Natur, das in vielen anderen Musikerzählungen Hoffmanns eine

35 Ebd., S. 449.

36 Ebd., S. 450.

37 Ebd., S. 448.

38 Gotthilf Heinrich von Schubert, Die Symbolik des Traumes. Faksimiledruck nach der Ausgabe von 1814, Heidelberg 1968, S. 65. 
so bedeutende Rolle spielt, sei es die Inspiration des Komponisten: Keines von beiden wäre möglich, wenn die Sängerin nicht ermordet worden wäre. Kunst entsteht hier als die bzw. aus der klingende(n) Erinnerung an ein Verbrechen, das im Prozess der künstlerischen Inspiration immer wieder nachgestellt wird: Wurde der erste Musiker zum Mörder, so wird es auch der zweite, wenn seine musikalischen Eingebungen nicht nur den Mord des ersten zur Voraussetzung haben, sondern erneut ,Adern aufgehen' müssen, ${ }^{39}$ damit die Geisterstimme erklingt, die ihm das Material für die eigenen Kompositionen liefert. Später im Lehrbrief heißt es entsprechend, der Musiker - nun vollends in die Fußstapfen des geheimnisvollen Fremden getreten - müsse den Geist der Natur beschwören wie der Magnetiseur (d.h. der geheimnisvolle Fremde) den der Somnambulen (d. h. des Burgfräuleins), ${ }^{40}$ um sie zum Singen zu bringen und diesen Gesang dann als sein eigenes Werk festhalten zu können. Der Musiker Chrysostomus ist so in die Reihe derjenigen Künstlerfiguren Hoffmanns zu stellen, die aus dem Tod oder Verstummen der weiblichen Muse den Stoff für die eigene Erzählung ziehen, so etwa auch der reisende Enthusiast in seinem Verhältnis zu Antonie („Rat Krespel“), Bettina („Das Sanctus“) oder Donna Anna („Don Juan“). ${ }^{41}$ Aus produktionsästhetischer Perspektive erscheint der Mord darum auf problematische Weise gerechtfertigt bzw. wird nachträglich umgewertet: „[vom] dämonische[n] Mißbrauch [...] [zum] Aufschwung zum Höheren“, ${ }^{42}$ wie Kreisler zusammenfasst, und man darf hierin wohl abermals eine Referenz auf Schubert erkennen, der mit dem Impetus der moralischen Läuterung vom „Mißbrauch“ der ursprünglich göttlichen Stimme des Gewissens durch einen „bösen Dämon“ schreibt, ${ }^{43}$ welcher es dennoch nie vermocht habe, die „höhere Stimme“ ganz auszulöschen; Erinnerungen an „Bilder und Empfindungen der besseren Stunden und Handlungen“ könnten demnach „Führer zurück zu dem höheren Ursprung“ werden. ${ }^{44}$

39 Hoffmann, „Kreisleriana“, S. 452.

40 Vgl. ebd., S. 454.

41 In Anlehnung u. a. an die seit Elisabeth Bronfens Untersuchung Over Her Dead Body: Death, Femininity and the Aesthetic (1992) vertraute Figur gehen u. a. folgende Studien dieser Künstlerfigur bei Hoffmann nach: Gabriele Brandstetter, „Die Stimme und das Instrument. Mesmerismus als Poetik in E. T. A. Hoffmanns ,Rat Krespel““, in: Brandstetter (Hg.), Jacques Offenbachs „Hoffmanns Erzählungen“. Konzeption, Rezeption, Dokumentation, Laaber 1988, S. 15-39, insbes. S. 31 ff.; Christine Lubkoll, Mythos Musik. Poetische Entwürfe des Musikalischen in der Literatur um 1800, Freiburg 1995, S. 276 ff.; Corina Caduff, Die Literarisierung von Musik und bildender Kunst um 1800, München 2003, insbes. S. 261-300.

42 Hoffmann, „Kreisleriana“, S. 454.

43 Schubert, Die Symbolik des Traumes, S. 58 u. 60.

44 Ebd., S. $65 \mathrm{f}$. 


\section{Dichtung als akusmatische Stimme einer goldenen Vorzeit}

Caroline Welsh hat in einem Artikel zum Stimmungsbegriff an den Texten des ästhetischen Theoretikers und empirischen Psychologen Johann Georg Sulzer zwei Aspekte überzeugend herausgearbeitet, die für das Verständnis des akustischen Spuks in der Literatur um 1800 hilfreich sind.$^{45}$ Erstens: Sulzer steht am Beginn einer psychologischen Theoriebildung des Unbewussten. Er behandelt „,dunkle Gegenden der Seele‘, die dem Bewusstsein nicht oder nur schwer zugänglich seien“ und an deren Schwelle zum bewussten Seelenleben er die Stimmung als „diffuse emotionale Grundbefindlichkeit“ verortet, die im bewussten Seelenleben als ein Indiz für unbewusste Prozesse, als ein Hinweis, dass sich im Unbewussten „etwas zusammenbraut“, zu verstehen sei. ${ }^{46}$ Zweitens: Der Begriff der Stimmung ermöglicht es Sulzer, die emotionale Verfassung des Gemüts und deren klanglichen Ausdruck immer schon zusammenzudenken. Denn mit dem ursprünglich musiktheoretischen Begriff der Stimmung kann Sulzer an die alte Tonartenlehre anschließen, in der bestimmte Tonarten bestimmten Affekten entsprechen, an stimmphysiognomische Vorstellungen, nach denen sich an der Stimmung der Stimme die des Gemüts ablesen lässt, sowie an die nervenphysiologische Resonanztheorie, der zufolge die ,Saiten der Seele“ wie die (und auch mit denen) eines ge- oder verstimmten Musikinstruments resonieren. An diese Prämissen anschließend kann Sulzer die These formulieren, dass jede das Unbewusste indizierende Gemütsstimmung ihren eigenen Ausdruck hat, der sich im ,Ton' manifestiere. Dass Musik aus dem Unbewussten des Komponisten, des Musikers oder - die Resonanztheorie macht es möglich - des Hörers oder gar aus einem kollektiven Unbewussten künden soll, ist in der Folgezeit dann eine von zahlreichen Musikschriftstellern (man denke neben Hoffmann etwa an Wackenroder oder auch - willensmetaphysisch gewendet - an Schopenhauer) vertraute Annahme. Aus Welshs Ausführungen kann also zunächst festgehalten werden:

45 Caroline Welsh, „Zur psychologischen Traditionslinie ästhetischer Stimmung zwischen Aufklärung und Moderne“, in: Anna-Katharina Gisbertz (Hg.), Stimmung. Zur Wiederkehr einer ästhetischen Kategorie, München 2011, S. 131-155. Welsh bezieht sich insbesondere auf den Artikel „Ton (Redende Künste)“, in: Johann Georg Sulzer, Allgemeine Theorie der Schönen Künste, 2 Bde., Leipzig 1771-1774, Bd.2, S.1158, sowie auf „Erklärung eines psychologisch paradoxen Satzes: Daß der Mensch zuweilen nicht nur ohne Antrieb und ohne sichtbare Gründe sondern selbst gegen dringende Antriebe und überzeugende Gründe handelt und urtheilet“ [1759], in: Johann Georg Sulzer, Vermischte philosophische Schriften, Hildesheim/New York 1974 [ND der Ausg. 1773], S. 99-121.

46 Welsh, „Zur psychologischen Traditionslinie“, S.138f. 
Aus dem Ton spricht das Unbewusste. Kein Wunder also, dass sich die Geister akustisch zu erkennen geben!

Vielleicht weniger vertraut, aber für die hier verhandelte Fragestellung wichtiger, ist jedoch Sulzers Annahme, dass nicht nur dem musikalischen Ton, sondern mehr noch dem „Ton der Rede“ eine solche Funktion zukommt, zu dem „[i]n der Dichtung [...] neben dem Klang der Stimme ,alles [gehört], was wir recht sinnlich vom Charakter der Rede empfinden', in Prosodie, der Rhythmus - aber auch die verwendeten Metaphern, Redefiguren und Tropen. “47 Diese Perspektive auf den Ton der Rede soll im Folgenden weiter vertieft und dabei der Blick vom (individuellen) Unbewussten ab- und dem kollektiven bzw. kulturellen Gedächtnis zugewendet werden, das ja schon mit Hoffmanns Erzählung in den Blick gerückt ist. ${ }^{48}$ Liest man dichtungstheoretische Schriften um 1800, so fällt - knapp resümiert - die Forderung auf, dass Dichtung laut gelesen bzw. deklamiert werden solle; damit hängt auch die Wiederkehr der Vorstellung des Dichter-Sängers und Rhapsoden um diese Zeit zusammen, wie wir sie auch in Hoffmanns „Lehrbrief“ wiederfinden. Diese Forderung hat vielerlei Gründe, etwa didaktischer und wirkungsästhetischer Natur, auf die hier nicht im Einzelnen eingegangen werden muss. ${ }^{49}$ Für mich interessant ist im vorliegenden Zusammenhang nur ein Punkt, nämlich die - auch in sprachphilosophischen Schriften der Zeit anzutreffende -

47 Sulzer, [Art.] „Ton (Redende Künste)“, zit. nach Welsh, „Zur psychologischen Traditionslinie“, S. 136; Hervorh. N. G.

48 Der Begriff des kulturellen Gedächtnisses steht für „eine Gruppe identitätsstiftende Wissensbestände, die in Speichermedien oder symbolischen Formen bzw. Praktiken externalisiert werden“: „Beim kulturellen Gedächtnis geht es um Kollektives, das ausschließlich in der Vergangenheit seinen Wurzelgrund hat und erst von da auf die Zukunft ausstrahlt“, so D. Bering, [Art.] „Kulturelles Gedächtnis“, in: Nicolas Pethes u. Jens Ruchatz (Hg.), Gedächtnis und Erinnerung. Ein interdisziplinäres Lexikon, Reinbek 2001, S. 329-332, hier S. 329.

49 Zur Deklamationspraxis und -theorie um 1800 vgl. den Beitrag von Mary Helen Dupree im vorliegenden Band, darüber hinaus: Joh. Nikolaus Schneider, Ins Ohr geschrieben. Lyrik als akustische Kunst zwischen 1750 und 1800, Göttingen 2004; Johannes Birgfeld, „Klopstock, the Art of Declamation and the Reading Revolution: An Inquiry into One Author's Remarkable Impact on the Changes and Counter-Changes in Reading Habits Between 1750 and 1899“, in: Journal for Eighteenth-Century Studies 31.1 (2008), S. 101-117; Mary Helen Dupree, „From ,Dark Singing“ to a Science of the Voice: Gustav Anton von Seckendorff, the Declamatory Concert and the Acoustic Turn Around 1800“, in: Deutsche Vierteljahrsschrift für Literaturwissenschaft und Geistesgeschichte 86.3 (2012), S. 365-396; dies., „Sophie Albrechts Deklamationen. Schnittstellen zwischen Musik, Theater, und Literatur“, in: Rüdiger Schütt (Hg.), Die Albrechts - Erfolgsautor und Bühnenstar. Aufsätze zu Leben, Werk und Wirkung des Ehepaars Johann Friedrich Ernst Albrecht (1752-1814) und Sophie Albrecht (1757-1840), Hannover 2015, S. 353-368; Sean Franzel, Connected by the Ear: The Media, Pedagogy, and Politics of the Romantic Lecture, Evanston, IL 2013; Reinhart Meyer-Kalkus, Stimme und Sprechkünste im 20. Jahrhundert, Berlin 2001. 
Annahme, dass im Klang der Sprache die Erinnerung an eine goldene Vorzeit archiviert sei, in der orale Dichtung als natürliche Form der Kommunikation und Selbstvergewisserung einer Gemeinschaft fungierte. ${ }^{50} \mathrm{Um}$ dies $\mathrm{zu}$ illustrieren, seien hier drei kurze Beispiele angeführt.

Als ein erstes Beispiel lässt sich Johann Gottfried Herders Interesse an mündlicher Dichtung anführen, wie er es zum Beispiel in den Vorreden zu seinen Volksliedsammlungen artikuliert. In seiner Vorlesung „Ueber die menschliche Unsterblichkeit“ (1792) legt Herder ganz grundsätzlich nahe, Sprache überhaupt als Geisterrede zu verstehen. Denn: „Wir denken in einer Sprache, die unsre Vorfahren erfunden, in einer Gedankenweise, an der so viele Geister bildeten und formten [...] und uns damit den edelsten Teil ihres Daseins, ihr innerstes Gemüt, ihre erworbnen Gedankenschätze huldreich vermachten. “" ${ }^{51}$ Herder stellt den Philosophen und Dichter der Gegenwart also zum einen als Geisterseher und -hörer dar: „[W]ir sind mit uns selbst nicht allein; die Geister andrer, abgelebter Schatten, alter Dämonen [...] wirken in uns. Wir können nicht umhin, ihre Gesichte zu sehn, ihre Stimmen zu hören“. ${ }^{22}$ Zum anderen als Bauchredner, in dem die Stimmen verstorbener Denker wieder lebendig werden:

So gingen in uns als Jünglinge die Gedanken derer über, die am meisten auf uns gewirkt haben; ihre Töne flossen in uns, wir sahen ihre Gestalten, verehrten ihre Schatten [...]. [Was, N. G.] im dunkeln Grunde unsres Gedankenmeeres tot und begraben zu liegen [scheint]; zu rechter Zeit steigets doch hervor [...]; alles [...] ist da, daß es zum Leben geweckt werde [...]..53

In den Vorreden zu seinen Volksliedsammlungen (1778-1779) geht es Herder dabei um eine ganz besondere Erbschaft, die den organischen Zusammenhang von Dichtung und Gemeinschaft ebenso wie von Wort und Ton betrifft. Herder

50 Prägnant findet sich diese Annahme z. B. auch im frühromantischen Ursprachenmythos wieder, den Sabrina Hausdörfer stichwortartig zusammenfasst: „Ursprache als Zugang zum verlorenen und künftigen Paradies; [...] Analogie von Ursprache und Poesie (A. W. Schlegel, Görres); Ursprache/Poesie als Korrektiv der entfremdeten Allgemeinsprache; Novalis' Theoreme über Ursprache und Poesie: Bestimmung von Ursprache als Ausdruck eines integren Zusammenhangs von Ich und Welt; Musikalität, Kunstcharakter der Ursprache“. Sabrina Hausdörfer, „Die Sprache ist Delphi. Sprachursprungstheorie, Geschichtsphilosophie und Sprach-Utopie bei Novalis, Friedrich Schlegel und Friedrich Hölderlin“, in: Joachim Gessinger u. Wolfert von Rahden (Hg.), Theorien vom Ursprung der Sprache, 2 Bde., Berlin 1989, Bd. 1, S. 468-497, hier S. 468.

51 Johann Gottfried Herder, „Über die menschliche Unsterblichkeit. Eine Vorlesung“, in: Herder, Werke, Bd. 8: Schriften zu Literatur und Philosophie 1792-1800, hg. v. Hans Dietrich Irmscher, Frankfurt a. M. 1998, S. 203-219, hier S. 207.

52 Ebd., S. 209.

53 Ebd., S. 208 u. 209. 
formuliert hier, wie Johann Nikolaus Schneider ausführt, „eine Poetik [...], die das Wesen der Poesie jenseits der Schriftlichkeit sucht“ und in deren Zentrum der „Ton“ als „organische[r] Zusammenhang von Wort, Melodie und Vortrag“ steht. ${ }^{54}$ Einen ,Nachklang، dieses Tons findet Herder in den alten Volksliedern, und er sammelt sie, damit seine Zeitgenossen die klingende Ahnung einer Gemeinschaft bekommen können, deren „Blume der Eigenheit [...], seiner [des Volkes, N. G.] Sprache und seines Landes, seiner Geschäfte und Vorurteile, seiner Leidenschaften und Anmaßungen“ die Poesie war: „[W]as die ältern Geschichtschreiber von den alten Barden, und die mittlern Geschichtschreiber von den Würkungen ihrer Minstrels und Meistersänger so viel sagen, kann man, dünkt mich, hier [in diesen Liedern, N. G.] noch immer im kleinen Nachklange ahnden!“

Ähnlich ist auch August Wilhelm Schlegel in seinen „Briefen über Poesie, Silbenmaß und Sprache“ (1795) davon überzeugt, dass in der gegenwärtigen Sprache eine ursprüngliche Sprache archiviert sei, die die tönende Sprache der Jugendzeit des Menschengeschlechts gewesen sei, in der Kunst und Poesie, „,von der gütigen Natur selbst gepflegt und erzogen“, ${ }^{56}$ „,an allen Angelegenheiten des Lebens den wichtigsten Anteil“57 gehabt und die „Zeichen der Mitteilung und [das] Bezeichnete[ ]“ noch in einem „notwendigen Zusammenhang“ gestanden hätten. ${ }^{58}$ „Indessen liegt doch jene innige, unwiderstehliche [...] Sprache der Natur in ihnen [den gebildeten Sprachen, N. G.] verborgen. “59 Es ist die Aufgabe der Poesie, diese verborgene Sprache in der gegenwärtigen Sprache wieder zu offenbaren: „Der ist ein Dichter, der die unsichtbare Gottheit nicht nur entdeckt, sondern sie auch andern zu offenbaren weiß“. ${ }^{60}$ Das wiederum bedeutet, dass der Dichter bestrebt sein muss, seine Sprache wieder tönen zu lassen, „Gesang und gleichsam Tanz in die Rede zu bringen [...]. Dies hängt genau mit ihrem [der Poesie, N. G.] Bestreben zusammen, die Sprache durch eine höhere Vollendung zu ihrer ursprünglichen Kraft zurückzuführen“. ${ }^{61}$

54 Schneider, Ins Ohr geschrieben, S. $27 \mathrm{f}$.

55 Johann Gottfried Herder, „Volkslieder“, in: Herder, Werke, Bd. 3: Volkslieder, Übertragungen, Dichtungen, hg. v. Ulrich Gaier, Frankfurt a. M. 1990, S. 9-430, hier S. 18.

56 August Wilhelm Schlegel, „Briefe über Poesie, Silbenmaß und Sprache“, in: Schlegel, Kritische Briefe und Schriften, Bd. 1: Sprache und Poetik, hg. v. Edgar Lohner, Stuttgart 1962, S.141180, hier S. 148.

57 Ebd., S. 164.

58 Ebd., S. 145.

59 Ebd., S. 146.

60 Ebd.

61 Ebd., S. 148. 
Das dritte, etwas ausführlichere Beispiel führt mich zu E. T. A. Hoffmann zurück. Die Erzählung „Die Automate“ (1814) enthält ein längeres, stark auf Gotthilf Heinrich von Schuberts Ansichten von der Nachtseite der Naturwissenschaft (1808) rekurrierendes Gespräch über die geheimnisvolle ,Naturmusik‘ ${ }^{62}$ Auch hier geht es darum, aus gegenwärtigen Klängen Rückschlüsse auf eine ferne Vorzeit zu ziehen, in der Natur und Mensch noch in einer allumschließenden Harmonie - durchaus auch im musikalischen Sinne verstanden - lebten: „In jener Urzeit des menschlichen Geschlechts, als es [...] in der ersten heiligen Harmonie mit der Natur lebte, [...] da umfing sie [die Natur, N. G.] den Menschen wie im Wehen einer ewigen Begeisterung mit heiliger Musik, und wundervolle Laute verkündeten die Geheimnisse ihres ewigen Treibens. “63 Der gegenwärtige Mensch vermag in manchen Naturphänomenen noch eine Ahnung von dieser Musik zu erhalten: „[N]och sind jene vernehmlichen Laute der Natur [...] nicht von der Erde gewichen“. ${ }^{64}$ Hoffmanns Musiker Ludwig jedoch geht es in diesem Zusammenhang darum, ein Instrument ähnlich der Glasharmonika zu imaginieren, das diese Naturlaute nachahmen und eine ebenso intensive Wirkung auf den Hörer haben könnte.

Schubert aber, auf den Hoffmann in diesen Passagen wörtlich rekurriert, geht es in diesem Zusammenhang um den Ursprung der Sprache: Sprache als „höhere Offenbarung“ des Wesens der Natur oder auch des „ewigen Geists“. ${ }^{65}$ Bezogen auf Vorstellungen der Sphärenharmonie spekuliert Schubert: „Den Rhythmus der Bewegungen der Welten [...] habe der Mensch zuerst nachgesprochen. [...] Auf diese Weise sei die älteste Naturweisheit und die Sprache selber durch unmittelbare Offenbarung der Natur an den Menschen entstanden. “66 Diese Sprache war „metrisch, in Versen“, war - obwohl die erste - gerade nicht „die unvollkommenste“, sondern „die vollkommenste, reichste und doch einfältigste, die wohlklingendste und rythmischeste“. In der Gegenwart sei sie zwar verloren, dennoch findet auch Schubert ein Residuum dieser alten Sprache in der Dichtung der Gegenwart: „Hierin glich die Sprache der Vorwelt dem Dichten“. ${ }^{67}$ In der Ergänzung zur vierten Auflage (1840) wird er noch deutlicher: „Was nun besonders diese Ansicht von einer lebendigen und belebenden Kraft der leben-

62 E. T. A. Hoffmann, „Die Automate“, in: Hoffmann, Sämtliche Werke in 6 Bdn., Bd. 4: Die Serapions-Brüder, hg. v. Wulf Segebrecht, Frankfurt a. M. 2001, S. 396-427.

63 Ebd., S. 421.

64 Ebd., S. 422.

65 Gotthilf Heinrich von Schubert, Ansichten von der Nachtseite der Naturwissenschaft [1808], 4. Aufl., Dresden 1840, S. 36.

66 Ebd., S. 38.

67 Alle vier Zit. ebd., S. 36. 
digen Rede betrifft, so ist sie wohl [...] auf unmittelbare, nicht blos älteste und alte, sondern auch neue und neueste Erfahrung gegründet. " ${ }^{68}$ Angespielt wird hier unter anderem auf christlich-sprachmystische Praktiken, zu denen Schubert sich hier selbst bekennt: „Wer den Weg [worauf das Menschenwort wieder Tat, [...] Name wieder Sache geworden], selbst gegangen ist, der weiß, daß es so wahr ist. "69 Hoffmanns Ludwig will also in der Musik, Schubert in der dichterischen oder sakralen Sprache dem klingenden Ursprung wieder nahe kommen. ${ }^{70}$

Durch Schubert sensibilisiert, fällt nun aber auch im Gespräch in „Die Automate“ auf, dass Ludwig den Nachhall der alten Naturmusik nicht nur in den beschriebenen Naturlauten, sondern zunächst einmal in einer Sage hört: „Ein Nachhall aus der geheimnisvollen Tiefe dieser Urzeit ist die herrliche Sage von der Sphärenmusik, welche mich schon als Knabe [...] mit inbrünstiger Andacht erfüllte“. ${ }^{71}$ Insofern ist es auch hier die Dichtung, und zwar die mündliche Dichtung - aus der Sage hört man noch das Sagen heraus - kollektiven Ursprungs, die als klingendes Residuum („Nachhall“) des goldenen Zeitalters aufgerufen wird; aufgerufen zudem in einer mündlichen Kommunikationssituation, in der Ludwig seinen Freund Ferdinand durch sein Erzählen allererst mit der Naturmusik vertraut macht. Wenn sich im Anschluss an das Gespräch dann in der Tat ereignet, wovon Ludwig erzählt hat - die Naturmusik erklingt im Garten des geheimnisvollen Mechanikers X -, so lässt sich das auch so lesen, dass Ludwig sie mit seiner mündlichen Erzählung herbei beschworen hat: Wort wird Tat, wie Schubert formuliert.

68 Ebd., S. 255; Hervorh. N. G.

69 Ebd., S. $261 \mathrm{f}$.

70 Erwähnt sei, dass Schubert auch in dem oben erwähnten Kapitel zum „Versteckten Poeten“ aus der Symbolik des Traums (1968 [1814]) von einer „ursprünglichen Geistersprache“ (ebd., S. 57) - eine „Bilder- und Gestensprache“, „allgemein verständlich“ und „furchtbar laut (ebd., S. 64 f.) - spricht, in der sich dem Menschen ursprünglich die „Stimme Gottes“ (ebd., S.59) mitgeteilt habe über das „Organ des Gewissens“ (ebd., S. 57), die aber nun infolge der „großen Sprachenverwirrung“ (ebd.) durch den „bösen Dämon“ (ebd., S.60) missbraucht werde: „Wir vernehmen [...] durch jenes Organ eine Geistersprache, die sich zwar zum Theil derselben Vorstellungen bedient, als die ursprüngliche, aber diese in einem ganz anderen ungeheuer verschiedenen Sinne gebraucht, sie zu einem ganz entgegengesetzten Zwecke mißbraucht“ (ebd., S. 58). Die Geschichte des ,Sprachfalls‘ wird auf der psychologischen Ebene gespiegelt.

71 Hoffmann, „Die Automate“, S. 421. 


\section{Chrysostomus als Tancredi, oder: Die Wiederkehr der Erinnerung}

Wenn es also der Ton der Dichtung ist, über den die Vorwelt in die Zeit der Lebenden hineinreicht, mehr noch: Wenn Sprache wesentlich Nach-Hall, akustisches Erinnern des Vergangenen ist, dann verwundert es - um auf die musikalische Heimsuchung im Lehrbrief zurückzukommen - nicht, dass die Geister der Vergangenheit primär sonal mit den Lebenden kommunizieren. Zumal auch im „Lehrbrief“ auf den Topos der geheimnisvollen ,Sprache/Musik der Natur` verwiesen wird, auf die unten noch einzugehen sein wird. Zugleich verhält es sich auch hier so, dass eine Szenerie mündlichen Erzählens gleich mehrfach aufgerufen wird, um die Geschichte der akustischen Heimsuchung zu erzählen: Die Erzählung besteht aus einem Brief des alten Kreisler an den jungen Kreisler, in den die mündliche Erzählung des Chrysostomus eingelassen ist, in die wiederum die Erzählung von Chrysostomus' Vater eingelassen ist, in der wiederum von Erzählungen und Liedern eines Fremden erzählt wird. Darüber hinaus ist in Chrysostomus' Erzählung auch noch die Rede von einem Lied, das der Vater ihm fast täglich vorgesungen hat und das den Jungen an den Moose-Stein hat denken lassen; und schließlich erwähnt Chrysostomus auch noch ,wunderliche Erzählungen' der Mutter, die ihm beim Anblick des Moose-Steins immer wieder in den Kopf kamen. Die Suggestionskraft des mündlichen Erzählens und Singens wird hier überdeutlich beschworen: Der Geist des toten Fräuleins taucht zuerst aus den Erzählungen und Liedern der Eltern auf, und der Musiker Chrysostomus gibt ihn mit seiner Erzählung an den alten Kreisler weiter, der ihn an den jungen Kreisler weitergibt, um ihm eine Lehre über das Wesen der Musik (und der Erzählung, wie noch zu zeigen sein wird) zu erteilen.

Und doch ergeben sich einige signifikante Differenzen zu den Theorien, die Dichtung als akusmatische Stimme einer goldenen Vergangenheit verstehen wollen, und diese Differenzen erlauben es, Hoffmanns Text als skeptischen Kommentar auf diese zu lesen. Der innerste Kern der ineinander verschachtelten Erzählungen spielt zwar auch in einer "fremden fabelhaften Zeit“, ${ }^{72}$ in der Dichtung und Musik noch nicht voneinander getrennt waren und deren Lieder sich durch das Ineinanderfallen von „Sprache“ und „Tönen“, ${ }^{73}$ von Bedeutung und Klang auszeichneten. Sie werden darum auch mit der Ursprungssprache der Engel verglichen, die wie die Lieder des fremden Rhapsoden ,geheimnis-

72 Hoffmann, „Kreisleriana“, S. 453.

73 Ebd. 
volle Dinge“,verständlich“ ausspricht, obwohl ihre Sprache dem Hörer „unbekannt" und „ohne Worte“ ist. ${ }^{74}$ Zugleich wird mit dieser fabelhaften Zeit auch die bereits angesprochene Idee einer Naturmusik/Natursprache verbunden. Die Rede ist von einem Wald „voll Ton und Gesang“, ${ }^{75}$ vom „Säuseln des Windes“ und dem „Geräusch der Quellen“, ${ }^{76}$ aus denen der Komponist Akkorde, Melodien und Harmonien heraushört: „,[D]ie Musik bleibt allgemeine Sprache der Natur, in wunderbaren geheimnisvollen Anklängen spricht sie zu uns “; ${ }^{77}$ und die Berufung des Musikers ist „das bewußtlose oder vielmehr das in Worten nicht darzulegende Erkennen und Auffassen d[ieser] geheimen Musik der Natur“" ${ }^{78}$ Eine zentrale Rolle spielen dabei die aus dem Blut der Toten gewachsenen, auch als ,Speicher-Medien` verstehbaren Moose, über deren Figuren sich ein intertextueller, hier aber nicht weiter auszuführender Bezug vor allem zu Novalis (der u.a. auf die Physiker Chladni und Ritter zurückgreift) herstellen lässt. Novalis hatte solche arabesken Figuren der Natur als Chiffrenschrift verstanden, aus denen die ursprüngliche Musik/Sprache der Natur wieder herausgelesen bzw. zum Klingen gebracht werden kann. ${ }^{79}$ So erzählt Chrysostomus von dem „Stein, an dessen Moosen und Kräutern, die die seltsamsten Figuren bildeten, ich mich nicht satt sehen konnte. Oft glaubte ich die Zeichen zu verstehen“; ${ }^{80}$ und er dachte, ,jene Gesänge [...] wären in den Moosen des Steins wie in geheimen wundervollen Zeichen aufbewahrt". ${ }^{81}$ Und in der Tat gelingt es dem zum Musiker berufenen Jüngling, vermittelt über die Naturgeräusche, die Lieder und Erzählungen der

\footnotetext{
74 Ebd., S. 449.

75 Ebd., S. 448.

76 Ebd., S. 454.

77 Ebd.
}

78 Ebd., S. 453.

79 So schreibt Novalis in den Lehrlingen zu Sais (1802): „Figuren, die zu jener großen Chiffernschrift zu gehören scheinen, die man überall, auf Flügeln, Eierschalen, in Wolken [...], im Innern und Äußern der Gebirge, der Pflanzen, der Tiere, der Menschen [...] erblickt. In ihnen ahndet man den Schlüssel dieser Wunderschrift, die Sprachlehre derselben“ (Novalis, „Die Lehrlinge zu Sais“, in: Novalis, Werke, hg. v. Gerhard Schulz, 3. Aufl., München 1987, S. 95-198, hier S. 95). Zur Chiffrenschrift der Natur als „Klangfiguren einer romantischen Poetik“ im Ausgang von Novalis vgl. Welsh, Hirnhöhlenpoetiken, S. 205-252. Zu Novalis’ Rückgriff auf Chladnis Klangfiguren und deren Fortschreibung bei Ritter vgl. Bettine Menke, „Töne-Hören“, in: Joseph Vogl (Hg.), Poetologien des Wissens um 1800, München, S. 69-95. Zu Arabesken der Natur bei Hoffmann (aber hier mit Bezug auf den „Goldnen Topf“) vgl. Günter Oesterle, „Arabeske, Schrift, Poesie in E. T. A. Hoffmanns Kunstmärchen ,Der goldne Topf ““, in: Athenäum. Jahrbuch für Romantik 1 (1991), S. 69-107.

80 Hoffmann, „Kreisleriana“, S. 450.

81 Ebd., S. 451. 
Eltern und die Moos-Figuren, die Musik/Sprache der Natur wieder zum Klingen zu bringen und sie sich für die eigene Kunst anzueignen: „[A]us einer fremden fabelhaften Zeit [trat] die hohe Macht in sein Leben, die ihn erweckte! - Unser Reich ist nicht von dieser Welt, sagen die Musiker“. ${ }^{82}$

Doch die fabelhafte Zeit ist im „Lehrbrief“ keine des organischen Zusammenhangs von Musik und Gemeinschaft, sondern eine der Zerstörung der Gemeinschaft durch die klingenden Erzählungen eines Fremden, die den Sündenfall d. h. sowohl die Erkenntnis (zum Beispiel die Unterscheidung von eigen/fremd, von gut/böse, die Entdeckung neuer Welten) als auch die Sexualität - in die Gemeinschaft tragen. Und die Natur hebt in dieser fabelhaften Zeit auch erst zu singen an als Reaktion auf den Mord, den der Rhapsode an dem Burgfräulein verübt. Als akusmatische Stimme gemahnt sie die Nachfahren an das Verbrechen, ist Ausdruck des kollektiven Traumas, das von Generation zu Generation weitererzählt wird, ohne dass jemals das Geheimnis rund um das Geschehen die traumatische Erinnerungslücke - wirklich gelöst werden könnte. Es ist, um an Caruths Tasso-Lektüre zu erinnern, eine „voice that cries out from the wound“. ${ }^{83}$

In der Tat sind die Gemeinsamkeiten zwischen Tassos epischer Episode und Hoffmanns in den „Lehrbrief“ eingebetteter „fabelhaft[er]“ Erzählung erstaunlich. ${ }^{84}$ Chrysostomus tritt gewissermaßen in die Fußstapfen Tancredis: Beide bewegen sich in einem Wald, in dem es akustisch spukt, weil die Seelen Verstorbener in ihm hausen - bei Tasso ist die Rede vom Brausen, Sausen, Stöhnen, Ächzen, Brüllen, Zischen, Heulen, Seufzen, Krächzen, Dröhnen sowie auch von „eine[m] Laut [...], kläglich, / Wie Seufzen und wie Schluchzen eines Menschen“ und vom „Schmerzens-Ach“, das aus den beseelten Zweigen und Stämmen dringt, in welche die Seelen der Toten eingewandert sind. ${ }^{85}$ Sowohl Tancredi als auch Chrysostomus treffen dann auf einen „herrlichen Baum“, ${ }^{86}$ an bzw. unter dem geheimnisvolle Chiffren erkennbar sind. Bei Tasso handelt es sich um ägyptisch anmutende Hieroglyphen (eines der Vorbilder für die um 1800 beliebten Chiffrenschriften) und um „[arabische] Zeichen“, die ihn mahnen, die Seelen im Tal des Todes nicht zu stören. ${ }^{87}$ Doch Tancredi kann, wie zunächst auch Chrysostomus, deren „[v]erborgene[ ] Bedeutung“ nicht ersinnen. ${ }^{88}$ Clorindas Stimme lässt sich erst hören, nachdem Tancredi den Baum verletzt hat und Blut aus der

82 Ebd., S. 453.

83 Caruth, Unclaimed Experience, S. 3.

84 Hoffmann, „Kreisleriana“, S. 449.

85 Tasso, „Die Befreiung Jerusalems“, S. 486.

86 Hoffmann, „Kreisleriana“, S. 448.

87 Tasso, „Die Befreiung Jerusalems“, S. 485.

88 Ebd., S. 486. 
Rinde fließt, das ringsum das Erdreich rötet; bei Hoffmann sind es die aus dem Blut gewachsenen roten Moose, aus denen der Gesang der Toten herausgehört wird; und die „roten Adern des Steins“ ,gehen auf“, beginnen gewissermaßen abermals zu bluten, als die Gestalt der Toten und ihr Gesang daraus ,empor fahren' ${ }^{89}$ Während sich für Tancredi damit sein individuelles Trauma wiederholt, wiederholt Chrysostomus gleichsam den Tötungsakt, der seit Generationen im kollektiven Gedächtnis seines Volkes verankert ist, und tritt damit das Erbe des fremden Rhapsoden an.

Die Gemeinsamkeiten zwischen beiden Erzählungen enden jedoch hier. Tassos mag man als literarische Urszene des traumatischen Wiederholungszwangs lesen; Hoffmanns macht daraus aber eine Ursprungstheorie der Musik/ Dichtung, durch die das Mordgeschehen, wie oben bereits geschildert, seine moralisch zweifelhafte Rechtfertigung erfährt. Hoffmann entwirft hier ein konsequent sentimentalisches Dichtungskonzept, das im Besingen der heilen Vorwelt immer schon die Notwendigkeit erkennt, diese Vorwelt verloren und, schärfer noch, selbst vernichtet zu haben - die Rückseite des ,janusgesichtigen ${ }^{60}$ Chry-

89 Hoffmann, „Kreisleriana“, S. $452 \mathrm{f}$.

90 Vgl. Schubert, Symbolik des Traumes, S.69, der hier von der Janusgesichtigkeit des inneren Poeten schreibt. Und zwar ist die Vernichtung als Konsequenz der Bewusstwerdung, des Unterscheidungen-Vornehmens, des Erkenntnisgewinns zu verstehen, die mit der Sprache und den Erzählungen des Rhapsoden in die ursprüngliche Gemeinschaft gekommen sind. Es handelt sich hierbei um eine fundamentale philosophische Denkfigur der (Früh-)Romantik. Hölderlin schreibt z. B. über das Urteil (womit sowohl der Erkenntnisakt als auch eine in der sprachlichen Form eines Satzes ausgedrückte Behauptung gemeint ist): „Urteil. ist im höchsten und strengsten Sinne die ursprüngliche Trennung des in der intellektualen Anschauung innigst vereinigten Objekts und Subjekts, diejenige Trennung, wodurch erst Objekt und Subjekt möglich wird, die UrTeilung. Im Begriffe der Teilung liegt schon der Begriff der gegenseitigen Beziehung des Objekts und Subjekts aufeinander, und die notwendige Voraussetzung eines Ganzen wovon Objekt und Subjekt die Teile sind“ (Friedrich Hölderlin, „Urteil und Sein“, in: Hölderlin, Sämtliche Werke und Briefe in 3 Bdn., Bd. 2: Hyperion. Empedokles. Aufsätze. Übersetzungen, hg. v. Jochen Schmidt, Frankfurt a. M. 1994, S. 502-503, hier S. 502). Die Sehnsucht nach dem heilen Ganzen, das für die Ursprungsmythen und Erinnerungskonzepte der Zeit prägend ist, hat diese unvermeidliche UrTeilung also immer schon zur Voraussetzung. Ruth Johnson schreibt dazu: „Romantic depictions of origin and of remembering aim to memorialize and to reconstruct aesthetically the fundamental moment of loss, or original trauma, or of separation. The Romantic term for this moment of separation is ,Ursprung ‘ or ,Urhandlung ${ }^{\text {* }}$ - the initial split between feeling and reflection, between subject and object - the first separation of the individual from the infinite" (Laurie Ruth Johnson, The Art of Recollection in Jena Romanticism: Memory, History, Fiction, and Fragmentation in Texts by Friedrich Schlegel and Novalis, Tübingen 2002, S. 1). Normalerweise wird in den (früh-)romantischen Konzepten der Dichter dann als derjenige idealisiert, der den Weg zurück in diese Einheit weisen kann; Hoffmann macht mit seinem fremden Dichter-Sänger aber darauf 
sostomus ist gleichsam der fremde Sänger. Zugleich macht Hoffmann deutlich, dass die Geisterstimmen, die dann zur Sprache kommen, immer schon Ergebnis des literarischen Imaginären, sei es des Dichters oder seines Lesers, sind. Jean Pauls Einsicht, dass die Erinnerung (im Unterschied zum Gedächtnis) immer schon ein schaffendes Vermögen ist, ${ }^{91}$ wird hier auf ihr poietisches Potenzial hin untersucht.

\section{Sonale Phantasien}

Eine weitere, auf den ersten Blick recht unscheinbare, aber auf den zweiten umso bedeutsamere Differenz zu Tassos Epos liegt nämlich darin, dass in Hoffmanns Erzählung die akusmatische Stimme der Toten gerade nicht laut wird. Bei Tasso ist Clorindas Rede als wörtliche Rede in die Erzählung eingelassen, Hoffmanns Erzählung aber bleibt konsequente Diegesis. Auch auf intradiegetischer Ebene affiziert ihr Gesang Chrysostomus gerade nicht, weil er ihn tatsächlich hören würde, sondern weil die suggestiven Erzählungen und Lieder seiner Eltern die sonale Phantasie des Hörers in Gang bringen. Chrysostomus' Hören der Geisterstimmen ist im „Lehrbrief“ zuverlässig mit einem träumerischen Zustand verbunden, in dem ihm, angeregt durch die Erzählungen und Lieder von Vater und Mutter, durch die Naturgeräusche und die Chiffrenschrift der Moose die Gesänge des toten Fräuleins aufgehen: „Wirklich geschah es auch, daß [...] ich [...] in hinbrütendes Träumen geriet und dann den herrlichen Gesang des Fräuleins vernahm“,92 „der Traum erschloß mir sein schimmerndes herrliches Reich“.93 Der Moment des Geisterhörens ist hier zugleich der der künstlerischen Inspiration, und künstlerische Inspiration erscheint als akustische Heimsuchung. Über den jungen Kreisler behauptet der alte Kreisler entsprechend, er halte die „Stimme“ des in seinem „Innern versteckten Poeten“ für eine von außen kommende (Geister-)Stimme. ${ }^{94}$ In seiner Adaption von Erzählungen Hoffmanns für

aufmerksam, dass dieser idealische ,Aufschwung، durch den Dichter immer schon die vorherige ,dämonische‘ Zerstörung durch denselben zur Voraussetzung hat. In jüngerer Zeit hat Derrida (in der Grammatologie) diese Einsicht breitenwirksam in der Figur einer ursprünglichen Gewalt (archi-violence) aufgegriffen und auf den Einsatz der Schrift (archi-écriture) bezogen, allerdings ohne dabei auf Hoffmann zu sprechen zu kommen.

91 Vgl. Oesterle, „Erinnerung in der Romantik. Einleitung“, S. 12.

92 Hoffmann, „Kreisleriana“, S. 451.

93 Ebd., S. 452.

94 Ebd., S. 448. 
Offenbachs Oper Les Contes d'Hoffmann (1881) hat der Librettist Jules Barbier diesen Moment anschaulich herausgestellt. Er verändert Hoffmanns Erzählung „Rat Krespel“ (1818) dergestalt, dass er die Figur eines dämonischen Arztes - Dr. Mirakel - einfügt, der die Sängerin Antonia durch seine suggestive Erzählung allererst dazu bringt, die Stimme der toten Mutter zu imaginieren, welche sie zum verbotenen (und für sie tödlichen) Singen inspiriert: Dr. Mirakel: „Ecoute! [...] C'est ta mère, c'est elle [...] son âme t'appelle / Comme autrefois [...] entends sa voix!“ Antonia: „Ma mère! [...] J’entends sa voix!“955

Als sonale Phantasie verstehe ich also Imaginationen, die durch suggestive mündliche Erzählungen ausgelöst werden und gegebenenfalls auch akustische Phänomene zum Gegenstand haben. Wie im Folgenden gezeigt werden soll, spielt für ihre Entstehung die Unbestimmtheit (visuell, semantisch) des Klanglichen eine zentrale Rolle. In der Musikästhetik um 1800 ist zum Beispiel eine Variante der sonalen Phantasie sehr präsent, die sich an Instrumentalmusik knüpft. Die Musikästhetiker dieser Zeit gehen nämlich davon aus, dass diese Musik aufgrund ihrer semantischen Unbestimmtheit dazu neigt, die Phantasie des Hörers in eine unwillkürliche und anarchische Tätigkeit zu versetzen. ${ }^{96}$ Forkel klagt zum Beispiel, dass „wir [...] häufig mit musikalischen Werken heimgesucht werden, wo eigensinnige aus einer zügellosen Phantasie entstandene Einfälle blos willkührlich [...] aufeinander folgen“, sodass der Hörer dazu verführt wird, sich „in die unsichern Gegenden einer wilden Einbildungskraft zu wagen““ ${ }^{97}$ Diese Gegenden werden häufig als Labyrinthe oder Irrgärten beschrieben, und die strukturlose Struktur und den sinnleeren Sinn ihrer Bilderfolgen versucht man mit dem Begriff der Arabeske zu fassen. Einschlägig ist dafür abermals E. T. A. Hoffmann, der zum Beispiel in „Beethovens Instrumental-Musik“ Kreislers Hören von Beethovens Klaviertrio op. 70 wie folgt beschreibt und dabei von der negativen Bewertung Forkels abweicht, indem er die Inspirationskraft dieser Hör-Erfahrung betont: „[W]ie einer, der in den mit allerlei seltenen Bäumen, Gewächsen und wunder-

95 Trio des 3. Akts; Jacques Offenbach, Hoffmanns Erzählungen. Phantastische Oper in fünf Akten [Les contes d'Hoffmann], quellenkrit. Neuausg. v. Fritz Oeser, dt. Übertr. v. Gerhard Schwalbe, Klavierauszug u. Vorlagenbericht, Kassel 1977-1981, hier Klavierauszug auf S. 229-231.

96 Vgl. ausführlich Gess, Gewalt der Musik, S. 253-261. Zum Begriff der Phantasie: Kant unterscheidet zwischen einer Einbildungskraft, die willkürlich Einbildungen reproduziert, und einer, die unwillkürliche Einbildungen hervorbringt und die er Phantasie nennt; vgl. Immanuel Kant, „Anthropologie in pragmatischer Hinsicht“, in: Kant, Werke in 6 Bdn., Bd. VI: Schriften zur Anthropologie, Geschichtsphilosophie, Politik und Pädagogik, hg. v. Wilhelm Weischedel, Darmstadt 1998, S. 395-690, hier S. 466.

97 Johann Nikolaus Forkel, Musikalisch-kritische Bibliothek, 3 Bde., Gotha 1778-1779, Bd.1, S. XIVf. 
baren Blumen umflochtenen Irrgängen eines fantastischen Parks wandelt und immer tiefer und tiefer hineingerät, [vermag ich] nicht aus den wundervollen Wendungen und Verschlingungen deiner Trios herauszukommen“. ${ }^{98}$ Hoffmann bezeichnet das Klaviertrio in seiner Rezension auch als ,ein freies Spiel der aufgeregtesten Phantasie“, das den Hörer ,in lichten, funkelnden Kreisen um[fängt], und seine Phantasie, sein innerstes Gemüt entzünde[t]“".99

Das Konzept der sonalen Phantasie greift ebenso, wie oben bereits angedeutet, für die Literatur um 1800, insbesondere wenn sie als Ton-Kunst verstanden wird. ${ }^{100}$ Erneut spielt hier die Unbestimmtheit eine zentrale Rolle, und zwar zum einen die Unbestimmtheit im Sinne der mangelnden Präsenz dessen, wovon erzählt wird, zum anderen die Unbestimmtheit als mangelnde Präsenz dessen, der erzählt. Das soll anhand von Edmund Burkes poetologischen Überlegungen in seiner Theorie des Erhabenen und anhand der Poetik der gothic novel kurz erläutert werden, um im Anschluss auf Hoffmann zurückzukommen. Burke beschäftigt sich in der Philosophical Enquiry into the Origin of Our Ideas of the Sublime and Beautiful (1757), die in Deutschland (u. a. von Lessing, Mendelssohn, Nicolai, Herder, Garve, Kant und Schiller) nicht nur intensiv rezipiert wurde, ${ }^{101}$ sondern auch für die Poetiken der Schauerliteratur, zu der auch viele Texte Hoffmanns gerechnet werden, von großer Wichtigkeit war, unter anderem mit der Frage, wie

98 Hoffmann, „Kreisleriana“, S. $57 \mathrm{f}$.

99 E. T. A. Hoffmann, „Beethoven, Zwei Klaviertrios Op. 70 (September 1812 und Januar/Februar 1813)“, in: Hoffmann, Schriften zur Musik. Nachlese, hg. v. Friedrich Schnapp, Darmstadt 1963, S. 118-144, hier S. 138 u. 143.

100 Vgl. hierzu auch das Fragment von Novalis (Das Allgemeine Brouillon, Nr. 245), in dem ebenfalls die Unbestimmtheit der Musiksprache den Geist zu freier Tätigkeit anregt und von dort der Link zur Ursprungssprache der Menschen geschlagen wird, die ein Gesang war: „Über die allg[emeine] $n$ Sprache der Musik. Der Geist wird frey, unbestimmt angeregt [...] - das dünkt ihm so bekannt, so vaterländisch - er ist auf diese kurzen Augenblicke in seiner indischen Heymath. [...] Unsre Sprache - sie war zu Anfang viel musicalischer und hat sich nur nach gerade so prosaisirt - so enttönt. [...] Sie muß wieder Gesang werden“; Novalis: „Das Allgemeine Brouillon 1798/99“, in: Werke, Tagebücher und Briefe Friedrich von Hardenbergs, Bd. 2: Das philosophischtheoretische Werk, hg. v. Hans-Joachim Mähl, Darmstadt 1999, S. 473-720, hier S. 517.

101 Am Anfang dieser Rezeption stehen Lessing, Nicolai und Mendelssohn; denn Lessing macht die anderen beiden noch im Jahr des Ersterscheinens auf die Publikation aufmerksam, und im folgenden Jahr schreibt Mendelssohn einen unpublizierten Kommentar (für Lessing) und eine Rezension, die in der Bibliothek der schönen Wissenschaften und freyen Künste publiziert wird. Zur Kritik Mendelsohns, insbes. auch an Burkes Dichtungskonzept, vgl. Tomá Hlobil, „Two Concepts of Language and Poetry: Edmund Burke and Moses Mendelssohn“, in: British Journal for the History of Philosophy 8.3 (2000), S. 447-458. Sie differieren vor allem darin, dass Burke Dichtung nicht als Nachahmungskunst verstehen will und statt in die Nähe der Malerei in die Nähe der Musik rückt: Worte als Töne, die eher über sympathy als über mentale Bilder wirken. 
man am besten terror beim Rezipienten auslösen könne. Eine Grundvoraussetzung, die er dabei ausmacht, ist „obscurity“: „To make any thing very terrible, obscurity seems in general to be necessary. “102 Obscurity - zu deutsch: Unbestimmtheit, Unklarheit, Dunkelheit - ist bei Burke die wichtigste Voraussetzung zur Erzeugung von terror, weil sie das Schreckliche nicht fassbar werden lässt (bzw. die Nicht-Fassbarkeit es allererst schrecklich werden lässt), und weil sie die Einbildungskraft des Rezipienten in eine unendliche Beschäftigung (,power on the fancy“, „,approach towards infinity“) ${ }^{103}$ versetzt, in der sie sich das Schreckliche immer noch schrecklicher vorstellt, als es jemals gemalt werden könnte.

Diese Beobachtung Burkes ist für die Bedeutsamkeit der akustischen Heimsuchung in der (Schauer-)Literatur um 1800 zentral. Denn für die „obscurity“, die Burke hier beschreibt, ist der Ausfall des Sehsinns wesentlich: Kann man etwas sehend erfassen, verliert es an Unbestimmtheit und damit an Schrecken und umgekehrt: „,consider[ ] how greatly night adds to our dread, in all cases of danger“. ${ }^{104}$ Konsequenterweise bezieht sich ein wichtiges Beispiel, das Burke für die Erzeugung von terror durch Unbestimmtheit gibt, auf „sounds“, genauer: auf unspezifische Geräusche:

A low, tremulous, intermitting sound [...]. I have already observed, that night increases our terror more perhaps than any thing else; it is our nature, that, when we do not know what may happen to us, to fear the worst that can happen us; and hence it is, that uncertainty is so terrible [...]. Now some low, confused, uncertain sounds, leave us in the same fearful anxiety concerning their causes, that no light, or an uncertain light does [...]. ${ }^{105}$

Das unbestimmte Geräusch lässt es gewissermaßen Nacht um den Hörer werden, weil er nicht sehen, und das heißt, nicht bestimmen kann, was ihn erwartet Michel Chion hat eben diese Psychodynamik als wesentliches Charakteristikum der ,akusmatischen Stimme‘ gefasst. ${ }^{106}$ Als Spukereignis scheinen solche Geräusche darum geradezu prädestiniert zu sein, stammen doch auch ,ghosts and goblins“, wie Burke schreibt, aus derselben Region der Unbestimmtheit: Sie affizieren das Gemüt deshalb so stark, weil „none can form clear ideas [of them]“ “. ${ }^{107}$

Diese doppelte Unbestimmtheit des akustischen Spuks nun kann, folgt man Burke weiter, ausgerechnet Literatur besser als jede andere Kunstform ausspie-

102 Edmund Burke, A Philosophical Enquiry into the Origin of our Ideas of the Sublime and Beautiful, Oxford/New York 1990, S. 54.

103 Ebd., S. 58.

104 Ebd., S. 54.

105 Ebd., S. $76 \mathrm{f}$.

106 Chion, Audio-Vision: Sound on Screen, S. 63.

107 Burke, A Philosophical Enquiry, S. 54. 
len. Er argumentiert zum einen, dass Literatur als eine Ton-Kunst zu verstehen sei (Worte werden hier primär als Klänge bzw. Klangfolgen gedacht), die nicht über die Nachahmung und das Aufrufen von klaren Vorstellungsbildern wirke, sondern über die „sympathy“ mit den Affekten, die die von den Tönen bezeichneten - gemeint ist ein arbiträres, aber durch Gewohnheit naturalisiertes Zeichenverhältnis - Dinge auf das Gemüt der beteiligten Personen haben. Er argumentiert zum anderen, dass die Malerei der „clarity“ verpflichtet sei und über das Aufrufen von entsprechend bestimmten Vorstellungen auf den Betrachter wirke. Es sei daher für die Malerei unmöglich oder mindestens lächerlich, an sich obskure Gegenstände (wie Geistererscheinungen) abbilden zu wollen. Ganz anders die Literatur: Im Vergleich mit der Nachahmungskunst der Malerei ist sie defizitär, denn sie kann nur eine ,very obscure and imperfect idea of such objects [hier: Paläste, Tempel, Landschaften, N.G.]“ geben; ${ }^{108}$ aber dieses Defizit wird dort zur Überlegenheit, wo es darum geht, die Leidenschaften des Rezipienten zu erregen. Denn „great clearness helps but little towards affecting the passions“. ${ }^{109}$

\footnotetext{
So that poetry with all its obscurity, has a more general as well as a more powerful dominion over the passions than the other art. And I think there are reasons in nature why the obscure idea [...] should be more affecting than the clear. It is our ignorance of things that [...] excites our passions. ${ }^{110}$
}

In gewisser Weise lässt es also auch die Literatur Nacht um den Rezipienten werden, insofern sie ihm die Dinge nicht klar vor Augen stellt, sondern stattdessen über deren Unbestimmtheit und die dadurch angeregte „power on the fancy“"111 einmal mehr seine Affekte, insbesondere seine Ängste anspricht. Diese Überlegenheit der Literatur kommt insbesondere dort zum Tragen, wo es um die Beschreibung von Gegenständen geht, die an sich unbestimmt sind (als da wären übernatürliche Kreaturen oder Ereignisse, wie zum Beispiel Spukgeschehen) und sich der Malerei daher vollständig entziehen. Burkes Text ist voll mit solchen Beispielen aus Miltons Paradise Lost (1667), etwa „his description of Death“, dem „king of terrors“, die für Burke vor allem aus einem Grund so wirkungsvoll ist: „In this description all is dark, uncertain, confused, terrible, and sublime to the last degree“. 112

108 Ebd.

109 Ebd., S. 56.

110 Ebd., S. 57.

111 Ebd., S. 58.

112 Ebd., S. 55. 
In den späteren gothic novels wird diese Einsicht hervorragend umgesetzt. Ein Beispiel: In The Mysteries of Udolpho (1794) von Ann Radcliffe wird die Heldin immer wieder von Geräuschen aufgeschreckt, die wie Schritte, wie ein Klopfen, wie ein Atmen usw. klingen, in unregelmäßigen Abständen wiederkehren und in ihrer Herkunft und Bedeutung schwer einzuschätzen sind. Bald ist sie überzeugt, dass es sich um Spukgeräusche handeln muss, und Furcht und terror bestimmen ihre Gemütslage, wie ausführlich beschrieben wird, sodass der Leser entsprechend - über die sympathy - mit ihr mitfühlen muss. Die sympathetische Übertragung von terror wird im Übrigen auch im Roman selbst thematisiert, wenn die abergläubische Furcht einer Bediensteten die Heldin des Romans, wie es heißt, infiziert. Diese „infection“ findet statt auf dem Weg einer mündlichen Erzählung vom angeblichen Spuk im Schloss, die immer wieder durch die geheimnisvollen Geräusche selbst unterbrochen wird, welche bald von der Bediensteten und dann auch von der Heldin als Spukgeräusche interpretiert werden:

„Did you ever hear, ma'amselle, of the strange accident, that made the Signor lord of this castle?“ „What wonderful story have you now to tell?“ said Emily, concealing the curiosity, occasioned by the mysterious hints she had formerly heard on that subject. [...] „[...] - Holy Virgin! what noise is that? did not you hear a sound, ma'amselle?“ [...] Emily, whom Annette had now infected with her own terrors, listened attentively; but every thing was still, and Annette proceeded: „[...] - There, again!“ cried Annette, suddenly [...]. They listened, and, continuing to sit quite still, Emily heard a low knocking against the wall. It came repeatedly. Annette then screamed loudly, and the chamber door slowly opened. ${ }^{113}$

Literatur operiert also in der Region der Unbestimmtheit, in der der Leser nicht auf klare Vorstellungen setzen kann, sondern im Wesentlichen auf seine affektiven Reaktionen verwiesen ist, die sowohl durch die unbestimmten Dinge selbst bzw. das dadurch in Gang gesetzte haltlose Tasten der Phantasie im Katastrophenmodus ausgelöst werden als auch durch ,sympathy' mit den Reaktionen der fiktionalen Charaktere. In der gleichen Region der Unbestimmtheit sind auch die Geister zu Hause sowie auch alle akustischen Phänomene unklarer Herkunft. Beide ahmt Literatur nicht nach (wie Malerei es - nach Burkes Überzeugung tun würde), sondern sie potenziert deren Unbestimmtheit durch die suggestive Unbestimmtheit ihrer sprachlichen Beschreibung. Im Fall der akustischen Phänomene ist dafür die Abwesenheit des Tons in der Diegese mitentscheidend: Weil er abwesend ist, muss der Text zu obskuren Beschreibungen greifen, die im Leser den Schrecken des Unbestimmten erregen. Ein illustratives Beispiel dafür bietet die sehr viel spätere, aber ebenfalls in der Tradition der gothic literature stehende

113 Ann Radcliffe, The Mysteries of Udolpho, London 1992, S. 236-239. 
novel of terror „Randolph Carter“ (1919) von H. P. Lovecraft, in der der „horror“, welcher den Überlebenden eines übernatürlichen Massakers in den Wahnsinn getrieben hat, eine vom Text unbeschreibbare, den Text schließlich sogar abbrechende Stimme ist - genauer: eine Telefonstimme aus dem Grab:

\begin{abstract}
And then there came to me the crowning horror of all - the unbelievable, unthinkable, almost unmentionable thing. [...] [I] heard the thing which has brought this cloud over my mind. I do not try, gentlemen, to account for that thing - that voice - nor can I venture to describe it in detail, since the first words took away my consciousness and created a mental blank [...]. ${ }^{114}$
\end{abstract}

Die Stimme erscheint hier als das Andere, das Abwesende des Textes. Wie das übernatürliche Monster seinen Schrecken dadurch gewinnt, dass es unsichtbar bleibt bzw. durch das Telefon nur partiell vermittelt wird, erhält die Stimme ihren Schrecken dadurch, dass sie unhörbar bleibt bzw. durch den Text nur ungenügend beschrieben wird. Die Stimme als das Monster des Textes: Welchen besseren Grund gäbe es, die Geister der Literatur akustisch spuken zu lassen! Der akustische Spuk erweist sich vor dem Hintergrund von Burkes Überlegungen als probate Wirkungsstrategie des literarischen Textes, der es auf den terror des Lesers anlegt.

\title{
Die akusmatische Stimme als narratives Verfahren
}

Kommen wir vor diesem Hintergrund zurück zu Hoffmann, der ein eifriger Leser von gothic novels in der Tradition von Walpole, Radcliffe und Lewis war, für die Burkes Überlegungen zur Erzeugung von „terror“ beim Leser von zentraler Bedeutung waren. ${ }^{115}$ Den „Lehrbrief“ (als zwölftes Stück der Kreisleriana im zweiten Teil der Fantasiestücke) publizierte Hoffmann im gleichen Jahr (1815)

114 H.P. Lovecraft, „Randolph Carter“, in: The H.P. Lovecraft Omnibus 1: At the Mountains of Madness and other Novels of Terror, London 1987, S. 353-360, hier S. 359. Der Text von Lovecraft deutet an, was ich hier auslasse: Einen großen Korpus von Erzählungen akustischer Heimsuchung, in denen die neuen akustischen Medien - hier das Telefon - eine zentrale Rolle spielen und in denen deren Unheimlichkeit thematisiert wird, übrigens unter Verwendung zahlreicher Topoi der früheren Schauerliteratur. Mein Text dürfte allerdings zeigen, dass die Narrative akustischer Heimsuchung keineswegs erst mit diesen neuen Medien zu ,boomen“ beginnen, sondern bereits in den Jahrzehnten davor prominent sind.

115 Radcliffe bezieht sich in ihrem poetologischen Essay „On the Supernatural in Poetry“ explizit auf Burkes Überlegungen zur Erzeugung von terror; vgl. Ann Radcliffe, „On the Supernatural in Poetry“, in: New Monthly Magazine 16 (1826), S. 145-152. 
wie Die Elixiere des Teufels, seinem eigenen, eng an Matthew G. Lewis' The Monk (1796) angelehnten Schauerroman, der wiederum durch Radcliffes The Mysteries of Udolpho inspiriert war. Insofern überrascht es wenig, in der schauerlichen Binnenerzählung des „Lehrbriefs“ manche Stilmittel der gothic novel umgesetzt zu finden, die schon Burke empfiehlt: so etwa „power“116 (bannende Wirkung des Fremden, Andeutung übernatürlicher Kräfte), „,suddenness“"117 (,plötzliches“ Verschwinden des Sängers) ${ }^{118}$ und „infinity“/,repetition“119 (alljährliches Nisten der Nachtigall, tägliches Singen um Mitternacht, Wiederholungszwang des Chrysostomus).

Besonders wichtig sind aber all jene Merkmale, die die Unbestimmtheit des Geschehens einerseits, die unbestimmte Beschreibung dieses Geschehens andererseits betreffen. Zum ersten Punkt gehört zum Beispiel der faszinierende, aber zutiefst ambivalente Gefühle auslösende Fremde, der von „unbekannten Ländern“ und „sonderbaren Menschen und Tieren“ zu erzählen weiß. ${ }^{120}$ Dazu gehört auch das wiederholt aufgerufene mitternächtliche Setting: die Treffen um Mitternacht, der Mord um Mitternacht, der Gesang der Nachtigall um Mitternacht. Sowie vor allem die akusmatischen Klänge (der von Ferne hinüberhallende Klang von Stimme/Laute; die Geisterstimmen aus dem Stein/im Baum). Hinzu kommt die Unbestimmtheit der Beschreibung. Wie der Fremde aussieht, erfährt man nicht, wohl aber, wie sein Aussehen auf die Betrachter wirkt: Er war „seltsamlich“ gekleidet, er kommt ihnen „wunderlich“ vor, man kann ihn nicht „ohne inneres Grausen“ anblicken. ${ }^{121}$ Was der Fremde erzählt, erfährt man ebenfalls nicht, sondern abermals nur, dass die Länder den Zuhörern „unbekannt“ und die Menschen „sonderbar“ sind, ebenso wie die Sprache des Fremden, in der er „unbekannte geheimnisvolle Dinge“ ausspricht. ${ }^{122}$ Indem die Beschreibungen also ganz und gar unbestimmt bleiben, zugleich aber die Wirkung des Fremden und seiner Erzählung auf die anderen Figuren geschildert wird, ergeht die Forderung an den Leser, sich seine eigene Vorstellung von einem Fremden zu machen, der ihm die gleichen „eiskalte[n] Schauer“, „folternde[ ] Angst“ und „Starrkr[ä] mpf[e] des Entsetzens“ bereiten würde wie den Figuren, mit denen er mitfühlt. ${ }^{123}$ Der Text selbst gibt diesen Rezeptionsmodus vor, indem er wiederholt von dem

116 Burke, A Philosophical Enquiry, S. 59-65.

117 Ebd., S. 76.

118 Hoffmann, „Kreisleriana“, S. 449.

119 Burke, A Philosophical Enquiry, S. 67-68.

120 Hoffmann, „Kreisleriana“, S. 449.

121 Ebd.

122 Ebd.

123 Ebd., S. 449 f. 
„dunkle[n] gestaltlose[n] Ahnen“ spricht, ${ }^{124}$ mit dem die Figuren auf den Fremden oder auf die Geisterstimmen reagieren - ein Ahnen, das Novalis auch als eine „ins Offene“, d. h. sowohl in die Imagination als auch in die Zukunft gerichtete Erinnerung verstanden hat. ${ }^{125}$ Der Binnentext, dessen Erzähler Chrysostomus ist, operiert also souverän mit dem Verfahren der Unbestimmtheit. Es betrifft auch die akustische Heimsuchung, insofern die Geisterstimme nie in direkter Rede erscheint, sondern immer nur vom Erzähler, auch in ihrer Wirkung auf den Hörer, beschrieben wird. Wie die Stimme klingt, ist somit der sonalen Phantasie des Rezipienten überlassen, der sie sich aufgrund ihrer Unbestimmtheit und seiner sympathy mit dem textimmanenten Hörer viel mysteriöser vorstellt, als sie jemals hätte hörbar werden können.

Aber darüber hinaus wirkt die Erzählung auch durch die Unbestimmtheit der Erzählerfigur: Die akusmatische Stimme wird hier in ein narratives Verfahren transformiert. Hoffmann überträgt gleichsam Goethes Einsicht, dass der Rhapsode „am allerbesten [hinter einem Vorhang]“ rezitieren solle, da sein Vortrag auf diese Weise umso intensiver wirken würde, in den Erzähltext, wenngleich mit ganz anderer Intention. ${ }^{126}$ Der „Lehrbrief“ weist Chrysostomus schon allein durch

124 Ebd., S. 449; vgl. auch ebd., S. 451.

125 Oesterle, „Erinnerung in der Romantik. Einleitung“, S. 8.

126 Johann Wolfgang von Goethe, „Über epische und dramatische Dichtung“, in: Goethe, Sämtliche Werke, Briefe, Tagebücher und Gespräche in 40 Bdn., Abt. I, Bd. 18: Ästhetische Schriften 1771-1805, hg. v. Friedmar Apel, Frankfurt a. M. 1998, S. 445-456, hier S. 447. Diesen Hinweis verdanke ich Reinhart Meyer-Kalkus, der mich während der Endredaktion meines Aufsatzes auf seinen noch nicht erschienenen Aufsatz aufmerksam gemacht hat, in dem er darauf eingeht: „Goethe als Vorleser, Sprecherzieher und Theoretiker der Vortragskunst“, erscheint in: Deutsche Vierteljahrsschrift für Literaturwissenschaft und Geistesgeschichte 90 (2016) [in Vorbereitung]. Auch Meyer-Kalkus spricht hier von der „akusmatische[n] Stimme des Erzählers“, die die „phantasiestiftende Kraft des dichterischen Wortes [...] entbinde“, jedoch liegt die Konzentration bei Meyer-Kalkus auf der Praxis des Vorlesens und seinem Mehrwert für die Rezeption von Literatur: „Bildschaffender als alle Medien wirkt der durchs gesprochene Worte angeregte innere Sinn der Zuhörer. Vorlesen ist insofern [...] eine der vornehmsten Kunstgattungen, weil sie die Spontaneität der Einbildungskraft des Zuhörers oder Betrachters wie keine andere aktiviert“. Bei mir geht es hingegen um die Transformation der akusmatischen Stimme in ein narratives Verfahren, das seine Wirksamkeit unabhängig davon entfaltet, ob der Text laut gelesen/vorgelesen wird oder nicht. Zudem sollten die Differenzen zwischen Goethes und Burkes akusmatischer Stimme nicht unterschlagen werden. Während Burke mit ihr auf die Unklarheit (auch der Vorstellung) und den dadurch erregten stärksten Affekt (des terror) setzt, erhofft sich Goethe vom unsichtbaren Rhapsoden eine „größere Klarheit des inneren Sinns“; Goethe steht dabei, wie Meyer-Kalkus zu Recht schreibt, in der Tradition der rhetorischen Enargeia-Lehre, nach der uns „Dinge und Vorgänge so plastisch und lebhaft vors innere Auge [geführt werden sollen], dass uns ist, als sähen wir sie leibhaftig vor uns“ (Meyer-Kalkus). In der gothic literature wird hingegen, ebenso wie in der 
seinen Namen als äußerst potenten Redner aus. Chrysostomus ist griechisch und heißt übersetzt „Goldmund“. ${ }^{127}$ Historisch lässt er sich auf den antiken Redner Dion Chrysostomos beziehen (ca. 40-120 n. Chr.), vor allem aber auf den Kirchenvater Johannes Chrysostomos (ca. 344-407 n. Chr.), Erzbischof von Konstantinopel, der als einer der größten christlichen Prediger gilt. Die besondere Potenz von Chrysostomus' Rede liegt nun nicht nur in seiner virtuosen Anwendung einer Rhetorik der Unbestimmtheit, sondern auch darin, dass diese Rhetorik den Erzähler selbst nicht ausnimmt. Denn Chrysostomus taucht im Text lediglich als akusmatische Stimme auf, und zwar als einzige wörtliche Rede des „Lehrbriefs“, die in die Erzählung des alten Kreisler eingelassen ist. Doch damit nicht genug, denn auch der alte Kreisler ist im Text nur als Stimme präsent, insofern er mit seinem Brief den jungen Kreisler direkt anspricht. Aus dem Brief tönt gewissermaßen die Rede eines Verstorbenen, der sich am Ende mit dem Zeichen des Grabeskreuzes verabschiedet. Sowohl bei Chrysostomus als auch beim alten Kreisler handelt es sich aber möglicherweise um bloße ,innere Stimmen' des jungen Kreisler, der im Text selbst nicht als Erzählinstanz auftaucht. Denn wie der alte Kreisler dem jungen Kreisler sagt, hat der junge Kreisler - um das Zitat abermals aufzugreifen - sein „Hörorgan so geschärft, daß [er] bisweilen die Stimme des in [s]einem Innern versteckten Poeten (um mit Schubert zu reden) vernimm[t], und wirklich nicht glaub[t], [er selbst] sei[ ] es nur, der gesprochen“. ${ }^{128}$ Der ,innere Poet" des jungen Kreisler: Das ist also - man denke an Schuberts „Janusgesicht[igkeit]“129 der inneren Stimme - entweder der als Geisterstimme in den Schüler eingegangene verstorbene Mentor oder Chrysostomus als geisterhafte Abspaltung des kreativen Persönlichkeitsteils des jungen Kreisler. ${ }^{130}$ In jedem Fall bleibt

romantischen Literatur, gerade nicht auf die innere Klarheit der Bilder, sondern auf das Dunkle, das Verworrene, die Ahnung und emotionale Erregung gesetzt. Goethe will ,Abstraktion“ und einen Schein von ,Allgemeinheit" durch die Unsichtbarkeit des Rhapsoden gewinnen (vgl. die bei Meyer-Kalkus vollständig zitierte Aussage); man kann sagen, sein Ideal ist die entkörperte Stimme; in der gothic literature geht es jedoch um die Unheimlichkeit des fehlenden Körpers, um den terror des Nicht-Wissens und um das Monströse der Abwesenheit. Interessant ist in diesem Zusammenhang auch die um 1800 wieder aufkommende Mode des unsichtbaren Chores, etwa in den Schiller-Gedächtnisfeiern; vgl. dazu Mary Helen Dupree, „Early Schiller Memorials (1805-1809) and the Performance of Literary Knowledge“, in: Dupree und Sean B. Franzel (Hg.), Performing Knowledge, 1750-1850, Berlin 2015, S.137-164.

127 Ich verdanke diesen Hinweis Britta Herrmann, für deren kritische Lektüre ich mich bedanke.

128 Hoffmann, „Kreisleriana“, S. 448.

129 Schubert, Die Symbolik des Traumes, S. 69.

130 Zur Möglichkeit einer solchen Abspaltung vgl. Schubert: „Nicht selten stellen sich, in der Bilder- und Gestaltensprache des Geistes, jene verschiedenartigen Stimmen, der Seele als besond- 
durch dieses Manöver (zwei akusmatische Erzählstimmen, für die drei mögliche Quellen infrage kommen) die für jeden Erzähltext zentrale Frage - wer spricht? unbeantwortbar. Die Erzählung spricht gewissermaßen mit mehreren Stimmen; in Hoffmanns Referenztext von Schubert findet sich die Monstrosität dessen in einem Beispiel wieder, „wo zu der Rede des Predigers der keine Arme hat, eine andere mit in sein Gewand versteckte Person die Gebärden macht, traurige, wenn jener fröhliche, fröhliche, wenn er traurige Worte spricht“. ${ }^{131}$ Auf diese Weise wird das ,gothische“ Motiv der Heimsuchung durch akusmatische Stimmen in ein narratives Verfahren transformiert, das auf die Selbsttätigkeit des Lesers zielt. ${ }^{132}$ Als akusmatische Stimmen setzen die Erzählstimmen dessen sonale Phantasie in Gang, die dann sowohl mögliche Quellen der Stimmen imaginiert, eine dämonischer als die andere, als auch im Raum des Imaginären die diversen Geisterstimmen laut werden lässt. Sie sind dann einerseits das vom Text heraufbeschworene Abwesende, die Stimme als ,Monster des Textes‘, als auch ,innere Stimme“ des Lesers, womit sowohl dessen poietische Kraft als auch - man denke an Schuberts Stimme des Gewissens - das individuelle oder sogar kollektive Unbewusste angesprochen ist.

\section{Coda}

Ausgehend von der gegenwärtigen Konjunktur des (immer wieder auf literarische Motive) zurückgreifenden Zusammendenkens von Musik und (traumatischer) Erinnerung habe ich eine literarische Vorgeschichte dieser Verbindung in Narrativen akustischer Heimsuchung um 1800 offengelegt, unter Konzentration auf eine Fallstudie von E. T. A. Hoffmanns „Kreislers Lehrbrief“. Dabei wurden fünf Thesen erarbeitet.

Erstens: Hoffmann formuliert in diesem Text einen auf einen ursprünglichen Gewaltakt gegründeten Musik-Entstehungs-Mythos, in dem Kunst als die bzw.

re, selbstständige Wesen dar, und der gute oder schlimme Dämon wird dieser wirklich sichtbar“ (Schubert, Die Symbolik des Traumes, S. 66). Bei Hoffmann werden sie nicht sichtbar, sondern als von außen kommend hörbar. Zu der These, dass Chrysostomus' Geschichte sich in Kreislers Leben wiederhole (bezogen sowohl auf die „Kreisleriana“ als auch auf Kreisler im „Kater Murr“) vgl. Alexis B. Smith, „Ritter’s Musical Blood Flow Through Hoffmann’s Kreisler“, in: John A. McCarthy et al. (Hg.), The Early History of Embodied Cognition, 1740-1920. The ,Lebenskraft'-Debate and Radical Reality in German Science, Music, and Literature, Leiden 2016, S. 145-162.

131 Schubert, Die Symbolik des Traumes, S. 70.

132 Ich danke Caroline Welsh für ihre hilfreichen Anregungen zu diesem Punkt. 
aus der klingenden Erinnerung an ein Verbrechen entsteht, das im Prozess der künstlerischen Inspiration immer wieder nachgestellt wird.

Zweitens: Dichtungstheorien um 1800 verstehen Dichtung als akusmatische Stimme einer goldenen Vorzeit, die im Klang der Sprache bzw. im Ton der Rede archiviert ist und in der Dichtung wieder laut werden soll.

Drittens: Hoffmann reflektiert diese frühromantische Sprachutopie kritisch, indem er danach fragt, was durch selbige verdrängt wird: die Komplizenschaft von Dichtung und (ur-teilender) ${ }^{\mathbf{1 3 3}}$ Aufklärung/Sprache.

Viertens: Aus Hoffmanns Text lässt sich das Konzept einer sonalen Phantasie gewinnen, die durch suggestive mündliche Erzählungen gespeist wird und/oder akustische Phänomene zum Gegenstand hat. Die Suggestionskraft der Erzählung basiert dabei auf einer - hier an Burke entwickelten - Rhetorik der Unbestimmtheit, die die gothic literature gezielt zur Erzeugung von terror beim Leser einsetzt. Diese Rhetorik bezieht sich sowohl auf die erzählten Inhalte, zu deren bevorzugten die akusmatische Stimme gehört, als auch auf die Diegese selbst, die die Dinge unsichtbar und unhörbar bleiben lässt.

Fünftens: Bei Hoffmann betrifft diese Unbestimmtheit auch die Erzählstimme selbst. Sein Text führt die Umwandlung des ,gothischen' Motivs der akusmatischen Stimme in ein narratives Verfahren - die Erzählstimme als akusmatische Stimme - vor, das auf die sonale Phantasie des Lesers zielt.

Die gegenwärtig populäre Idee einer musikalisch getriggerten Erinnerung demonstriert auf diese Weise die Wirkmächtigkeit eines literarischen Narrativs, das es nicht nur auf suggestive Erzählungen anlegt, sondern auch ein Bewusstsein von der Janusgesichtigkeit der sentimentalischen Sehnsucht nach dem Vergangenen mit sich führt. Über die musikalisch getriggerte Erinnerung lässt sich daraus entnehmen, dass es sich bei diesem Hör-Wissen immer schon (auch) um eine sonale Phantasie des Hörers handelt. Der Kurzschluss von Musik und Erinnerung profitiert demnach von der relativen Unbestimmtheit der Klangeindrücke ebenso wie von unserer emotional durchwirkten Imaginationslust. ${ }^{134}$ In diesem Sinne handelt es sich beim ,Hören der Erinnerung“ eher um ein produktives Ahnen der Vergangenheit denn um die Reproduktion eines Wissensbestandes, sodass sich das im vorliegenden Buch behandelte Hör-Wissen hier in besonderer

133 Erinnert sei hier noch einmal an Hölderlin und seine Rede von der „ursprüngliche[n] Trennung des in der intellektualen Anschauung innigst vereinigten Objekts und Subjekts“, der „UrTeilung“ (Hölderlin, „Urteil und Sein“, S. 502). Siehe dazu auch die obige Fußnote.

134 Matussek fasst diese Einsicht in seinen Überlegungen zum déjà entendu übrigens in die Formel: Vagheit/Ferne der Klangeindrücke plus Konfabulation des Hörers - daraus entstehe das erinnernde Hören; vgl. Matussek, „Déjà-entendu“, S. 302. 
Weise als prozessual und als einer historisch und subjektiv bestimmten Poiesis unterworfen erweist. 
Brought to you by | Universitaetsbibliothek Basel

Authenticated Download Date | 3/14/18 9:39 AM 